\title{
Modelo de Proceso de Conceptualización de Requisitos
}

\author{
Alejandro Hossian \\ Grupo de de Investigación en Sistemas Inteligentes Aplicados a Ingeniería \\ Facultad Regional Neuquén \\ Universidad Tecnológica Nacional \\ Neuquén, Argentina \\ alejandrohossian@yahoo.com.ar
}

\begin{abstract}
Resumen - El proceso de captura de requisitos constituye un proceso con connotaciones sociales relacionadas con diferentes personas (stakeholders), una circunstancia que hace que se presenten ciertos problemas cuando se lleva adelante la conceptualización de requisitos. En este trabajo se propone un Proceso de Conceptualización de Requisitos que se estructura en dos fases: (a) Análisis Orientado a al Problema: cuyo objetivo es comprender el problema dado por el usuario en el dominio en el que este se lleva a cabo, y (b) Análisis de Orientado al Producto: cuyo objetivo es obtener las funcionalidades que el usuario espera del producto de software a desarrollar, teniendo en cuenta la relación de estas con la realidad expresada por el usuario en su discurso. Se proponen seis técnicas que articulan cada una de las tareas que componen las fases de proceso propuesto.
\end{abstract}

Palabras Clave - Proceso de captura de requisitos, conceptualización de requisitos, proceso de conceptualización de requisitos, análisis orientado a al problema, análisis de orientado al producto, técnicas de modelado.

\section{INTRODUCCION}

En estadios tempranos de la Ingeniería de Requerimientos, Alford [1], Yeh y Zave [2] y Davis [3] identificaron la necesidad de obtener una representación intermedia de la información obtenida - conceptualización de los requisitos -, facilitando de esta manera una captura adecuada del problema a resolver por parte del profesional de ingeniería de software antes de pasar a la construcción de los modelos conceptuales, habida cuenta de que una correcta construcción de estos modelos es fundamental para el éxito en el desarrollo del proyecto software, mientras que su incorrección puede perjudicar seriamente a las organizaciones implicadas [4].

Asimismo cabe señalar, que la escasa existencia de trabajos referidos a la elaboración de representaciones intermedias de los caudales de información obtenidos a lo largo de la actividad de educción, tendientes a una búsqueda de reducción de la complejidad de la realidad y su problemática expresada por el cliente y/o usuario en su discurso, agravan aún más este problema.

En este sentido y en lo que se refiere a la gestión de requisitos en el campo de los sistemas de información, se pueden citar algunos principios fundamentales de estructuración de la información - "Partición, Abstracción y Proyección" -, los cuáles proporcionan una estructura de conocimiento a fin de contribuir a una visión simplificada de la realidad y su problemática [5]. Los elementos que suelen utilizarse para este análisis de problemas son los objetos, las funciones y los estados, pudiendo éstos describirse en múltiples niveles de detalle. Dado que hay tantas relaciones que pueden existir entre todos los elementos, se hace necesario disponer de una estructura de conocimiento (colección estructurada de conceptos y sus interrelaciones) que permita la captura estas relaciones.

A partir de la partición, es posible capturar la relación estructural "agregación/parte de" entre objetos, funciones o estados en el dominio del problema. A partir de la abstracción, es posible capturar las relaciones estructurales "general/específico" o "ejemplo de" entre objetos, funciones o estados en el dominio del problema. A partir de la proyección, es posible capturar la relación estructural "visión de" entre objetos, funciones o estados en el dominio del problema.

Si bien estos principios ofrecen su aporte a los efectos de precisar un mejor entendimiento de sus requisitos, son de carácter muy general y de poco nivel de detalle.

De igual manera y en lo que respecta a la gestión del conocimiento dentro del campo de los sistemas basados en conocimientos (SBC), se puede citar una técnica de representación intermedia como el "Análisis de Protocolos" [6]. Este método es de gran utilidad a los fines de obtener heurísticas que el experto utiliza en la solución de problemas, pero que le resulta difícil explicar [7].

En síntesis, esta técnica consiste en grabar en un protocolo el comportamiento del experto mientras este trabaja en la solución del problema. Luego ese protocolo se transcribe y se analiza para, finalmente, interpretarlo y convertirlo en un conjunto de razonamientos que convergen a la solución del problema. La reconstrucción de esta solución permite modelar los conocimientos del experto.

La forma más clásica de representar este conocimiento consiste en codificar el mismo en la forma de reglas de producción, las cuáles presentan una parte izquierda $[\mathrm{PI}]$ y una parte derecha [PD] (Si...[PI].. Entonces..[PD]..).

Cabe destacar, que si bien esta técnica permite poner en evidencia carencias y fallos en el documento de educción de conocimientos, también es cierto que determinados procesos no son reportados por el experto y que no todos los conocimientos son fáciles de representar en forma de reglas [8].

Se ha propuesto como objetivo de este trabajo definir un marco metodológico que incorpore una actividad de conceptualización tendiente a mejorar la comprensión y captura de requisitos de usuario en la fase de análisis de la Ingeniería de Requerimientos del Software. Esta actividad de conceptualización buscará plasmar en un esquema de 
representación integrado la realidad descripta por el cliente y/o usuario en su universo de discurso, así como también la problemática embebida en ella que se intenta resolver mediante una solución software.

La investigación se enfoca a plantear un proceso de conceptualización que funcione a modo de puente vinculando las actividades propias de la educción y el modelado de requisitos en la Ingeniería del Software. Con base en problemas de comunicación e interpretación entre clientes y/o usuarios y desarrolladores, surge la necesidad de una representación intermedia que facilite la consistencia del proceso de convergencia de los requisitos planteados por el usuario hacia los respectivos modelos conceptuales.

La investigación ha buscado formular contribuciones sobre: [a] actividades de conceptualización de requisitos de usuario capaces de proporcionar un mecanismo de análisis del discurso que permita al desarrollador relevar aquellos aspectos significativos de la realidad y su problemática, [b] mecanismos de derivación de esquemas de representación que faciliten la comprensión del problema de usuario y su asociación con aspectos de la solución software a implementar, y [c] estrategias que fortalezcan los canales de comunicación entre clientes, usuarios y desarrolladores a los efectos de optimizar la validez de las representaciones elaboradas para modelar la realidad y su problemática en función de su grado de aproximación al entorno del usuario.

Se presenta la Descripción del Problema (Sección II) en la que se señala la importancia que tiene la actividad de requisitos para la comprensión del problema de investigación, se caracterizan las actividades de educción de requisitos y de modelado conceptual, así como la forma en que se vinculan entre ellas para la construcción de los modelos conceptuales, se identifica el problema de investigación a partir de las dificultades provenientes del proceso de educción y como estas impactan en la construcción de los modelos conceptuales, se caracteriza el problema abierto y se concluye con un sumario de investigación.

Se propone una solución (Sección III) basada en: un modelo de proceso de conceptualización de Requisitos, del cual se abordan las cuestiones generales de mayor relevancia, se presenta la propuesta de dicho modelo y se explican en detalle las dos fases que componen el modelo (fase de Análisis Orientado al Problema y fase de Análisis Orientado al Producto), junto con las tareas que deben realizarse para llevar a cabo estas fases y los productos que se obtienen con la implementación de las mencionadas tareas. Se introducen las técnicas asociadas a las tareas del modelo de proceso. En primer término se presentan las técnicas utilizadas en la fase de Análisis Orientado al Problema como: la Técnica de Segmentación del Discurso de Usuario (TS - DU), las Técnicas Cognitivas de Identificación de Conocimientos Factuales, Procedurales, Contextuales y de Asociación (TCI CFPCA) y la Técnica de Construcción del Diagrama de Espacio Problema de Escenarios de Usuario (TCD - EPEU). En segundo término se presentan las técnicas utilizadas en la fase de Análisis Orientado al Producto como: la Técnica de Construcción del Diagrama de Escenarios de Usuario (TCD EU), la Técnica de Refinamiento del Diagrama de Escenarios de Usuario (TRD - EU) y la Técnica de Construcción del Diagrama del Mapa Unificado de Escenarios de Usuario (TCD - MUEU).
Finalmente se formulan conclusiones (Sección IV) se presentan las aportaciones y se destacan las futuras líneas de investigación que se consideran de interés en base al problema abierto que se presenta en este trabajo.

\section{DESCRIPCIÓN DEL PROBLEMA}

En esta sección se presenta la importancia que tiene la actividad de requisitos para la comprensión del problema de investigación (sección II.A), se caracterizan las actividades de educción de requisitos y de modelado conceptual, así como la forma en que se vinculan entre ellas para la construcción de los modelos conceptuales (sección II.B), se identifica el problema de investigación a partir de las dificultades provenientes del proceso de educción y como estas impactan en la construcción de los modelos conceptuales (sección II.C), se caracteriza el problema abierto (sección II.D) y se concluye con un sumario de investigación (sección II.E).

\section{A. La Actividad de Requisitos en la Comprensión del Problema}

En el proceso de desarrollo de software se pueden distinguir diferentes actividades que han de llevarse a cabo. De acuerdo al enfoque tradicional, el ciclo de vida del producto software supone un modelo en el cuál dicho producto evoluciona a través de una secuencia ordenada de transiciones de una fase a la siguiente conforme a una aproximación lineal o secuencial. En este sentido, el ciclo de vida del producto software se ha estructurado en las siguientes fases: Requisitos, Diseño, Implementación, Pruebas y Mantenimiento [3].

De las actividades citadas, cabe destacar que la actividad de Requisitos posee especial importancia en la construcción de un producto software, habida cuenta de que su principal objetivo consiste en identificar, entender y especificar las necesidades del usuario [9].

No obstante la poca uniformidad que se encuentra en la terminología empleada en las diferentes actividades relacionadas con los Requisitos [10], en el presente trabajo se utilizará la terminología propuesta por Davis [3], En este sentido, se entiende que la fase de Requisitos está compuesta por dos actividades que, aunque conceptualmente se establecen como diferentes, ambas se encuentran relacionadas:

1. Análisis del Problema cuya finalidad consiste en entender de manera precisa el problema que se ha de resolver y caracterizar la solución que este tiene.

2. Especificación de Requisitos cuya finalidad es crear un documento que constituye la especificación de requisitos del software, y en el cuál se describe con exactitud lo que el usuario espera del futuro producto software a desarrollar.

En el contexto de este lineamiento, el presente trabajo de investigación se enmarca dentro del proceso de "Análisis del Problema". Esta actividad resulta ser de vital importancia dentro del proceso de desarrollo, ya que como se indicara anteriormente, tiene como objetivo entender la necesidad del usuario y como ésta necesidad debe ser resuelta o satisfecha. Como consecuencia de este proceso, los desarrolladores se encontrarán en condiciones de abordar la construcción del sistema [11].

A modo de conclusión en lo que respecta al encuadre del problema de investigación dentro de la tarea de Análisis, conviene citar las palabras de Davis [3] en este sentido: 
"El Análisis es la actividad que incluye aprender acerca del problema a resolver [.....], entender las necesidades de los potenciales usuarios, tratar de identificar quien es realmente el usuario y entender todas las restricciones a la solución"

En el marco de esta cita, dos términos resultan ser de suma importancia: aprender y entender. El análisis constituye una actividad que pretende proporcionar al Ingeniero de Requisitos (IR) el conocimiento necesario para solucionar los problemas que surgen en un dominio determinado y que, habitualmente, le es ajeno.

En otras palabras, el conocimiento a adquirir es extraño para el IR, y sólo puede obtenerse mediante un aprendizaje in situ, es decir, a través de una inmersión en el dominio del problema. Dominio éste que pertenece a los usuarios, quienes son los encargados de vivenciar el problema al cual el Ingeniero de Requisitos (IR) debe darle una solución.

A partir del análisis es posible "identificar los conceptos relevantes del problema a resolver", como así también "entender las restricciones que debe cumplir cualquier solución software que le sea aplicable". En función del conocimiento adquirido en esta fase, el IR estará en condiciones de derivar una solución para el problema planteado por el usuario.

\section{B. Educción de Requisitos y Modelado Conceptual}

Conforme a la definición de Davis expresada en sección anterior, acerca del significado de la tarea de Análisis, caben distinguir, dentro del proceso por medio del cual se lleva a cabo esta tarea, dos subprocesos que resultan ser sustanciales para llevar a cabo con éxito la construcción de un producto software: "Educción de Requisitos" y "Modelado Conceptual". A continuación se proporciona una breve caracterización de estos subprocesos:

- Educción de Requisitos: este proceso tiene como objetivo adquirir el conocimiento del dominio de la organización usuario, de manera tal de que sea posible identificar los conceptos, relaciones y funciones más relevantes. Como consecuencia del desarrollo de este proceso se obtiene lo que se denomina Universo de Discurso, al cual se lo puede definir como aquella parte del mundo cuya información va a ser manejada por el sistema software a construir [12].

- Modelado Conceptual: este proceso tiene como objetivo la construcción de modelos que describen parte del mundo real de una forma no ambigua y no redundante [9], y que representan el problema y su solución [13]. Habida cuenta de que estos modelos permiten clasificar la información recolectada en el proceso de Educción, y por consiguiente, comprender mejor el problema en cuestión, a estos modelos se los denomina Modelos Conceptuales.

En virtud de lo expuesto, se estima conveniente hacer referencia a dos citas importantes del significado de los modelos conceptuales en relación con el universo de discurso: la primera señala que los modelos conceptuales constituyen una representación del universo de discurso en el cual tiene lugar el problema [14]; la segunda, por su parte, define a los modelos conceptuales como una descripción del universo de discurso haciendo uso del lenguaje y la forma de pensar de los expertos del dominio y de los usuarios [13]. La figura 1 ilustra el proceso de construcción de los modelos conceptuales a partir de la actividad de educción de requisitos.

\section{Identificación del Problema de Investigación}

En la sección anterior, han sido caracterizados cada uno de los procesos de Educción de Requisitos y Modelado Conceptual, así como también la vinculación que se establece entre ambos a partir de los productos que de ellos se generan (Universo de Discurso y Modelos Conceptuales).

A los efectos de identificar el problema que se pretende resolver en el presente artículo, se debe empezar por identificar los inconvenientes que presenta el primer eslabón del proceso macro que se ilustra en la figura 1; es decir el proceso de educción de requisitos. El proceso de educción, cuyo objetivo central consiste en dar a luz a los requisitos, no solo constituye un proceso de carácter técnico para construir un determinado sistema, sino también un proceso con importantes connotaciones de tipo social que involucra a distintas personas (stakeholders); circunstancia ésta que origina que se presenten ciertos problemas a la hora de la realización de dicho proceso de educción [15]. Asimismo, con respecto a los stakeholders cabe aclarar que dicho término se utiliza en referencia a cualquier persona o grupo que se verá afectado por el sistema en forma directa o indirecta; entre los mismos se pueden citar a usuarios finales que interactúan con el sistema, así como también a demás personas que pueden verse afectadas por la puesta en marcha del mismo (profesionales que proporcionan mantenimiento a otros sistemas relacionados, expertos en el dominio del sistema y gerentes de negocio, entre otros).

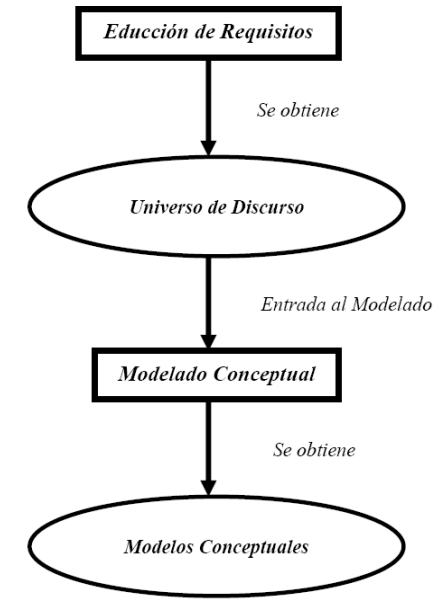

Fig. 1. Relación entre los procesos de Educción de Requisitos y Modelado Conceptual

Los problemas citados anteriormente pueden ser enfocados en función de los inconvenientes a los que se ven enfrentados los ingenieros de requisitos a la hora de relevar y comprender los requisitos que manifiestan los diferentes stakeholders [16], [17]. Estos problemas pueden ser sintetizados de la siguiente manera:

- En la mayoría de los casos los stakeholders desconocen lo que desean obtener del sistema informático, resultándoles difícil expresar cual es el problema que pretenden que sea resuelto $y$, en consecuencia, lo que deseen que haga el sistema.

- Por lo general, los stakeholders manifiestan sus requisitos con su propio lenguaje natural y con un conocimiento implícito de su propia labor. Por consiguiente, los ingenieros de requisitos, que en la generalidad de los casos carecen de la 
experiencia y el conocimiento en el dominio del usuario, deben comprender en forma correcta estos requisitos.

- Muy posiblemente, los diferentes stakeholders involucrados en la construcción del sistema posean diferentes requisitos, los cuales pueden ser expresados de varias formas distintas. Por consiguiente, los ingenieros de requisitos deben tener en consideración todas las posibles fuentes potenciales de requisitos y hallar coincidencias y conflictos.

- También es posible que factores de carácter político tengan cierta influencia en los requisitos del sistema. A modo de ejemplo, un director de un cierto departamento puede solicitar requisitos del sistema a los efectos de tener mayor influencia en el seno de la organización.

Continuando en esta línea, por las razones expuestas se puede afirmar que el proceso de educción es difícil de llevar a cabo. En este sentido, conforme a Christel [18] y con idea de complementar los problemas expresados anteriormente, se estima conveniente añadir las siguientes consideraciones:

- Mucha información importante para la construcción del producto software no llega a ser verbalizada, quedando así plasmados importantes huecos en la información capturada.

- En la mayoría de los casos el proceso de educción se lleva a cabo en forma pasiva en relación con el cliente y/o usuario, cuando en realidad debe ser afrontado en forma cooperativa.

Ahora bien, en virtud del conjunto de limitaciones a las que hacen mención Sommerville y Christel, propias del proceso de educción, es que surge la necesidad de explorar y analizar aquellas particularidades que son inherentes a este proceso $\mathrm{y}$ que, en tal sentido, contribuyen a caracterizarla.

Caracterizada la tarea de educción, se infiere que el eje de la misma se focaliza en la comunicación que se establece entre el usuario y el IR. Este, cuando desarrolla su trabajo de educción, debe capturar y modelar una realidad que enmarca una problemática, y cuya solución, debe ser abordada a través de un producto software. Siendo esta realidad un elemento intangible y, por lo general también compleja, es que también resulta difícil su captura.

Ahora bien, la captura de esta realidad junto con su problemática quedan plasmadas en el discurso del usuario, a partir del cuál el IR debe confeccionar el universo de ese discurso ("situaciones, hechos, objetos, etc., en los que se focaliza el estudio durante la educción y que, en consecuencia, resultan ser sustanciales a la hora de abordar el desarrollo del futuro sistema software [12]"), a los efectos de poder alcanzar así los modelos conceptuales ya en la fase de análisis de requisitos.

Estos inconvenientes, propios del proceso de educción, hacen que se dificulte la elaboración del universo de discurso por parte del IR, así como también la construcción de modelos conceptuales adecuados [19], [20]; es decir que estos problemas, que comienzan a manifestarse en el proceso de educción de requisitos y a partir de la comunicación entre el usuario y el ingeniero, seguramente se propagarán en la actividad de construcción de los modelos conceptuales. Estos inconvenientes confluirán, de manera inexorable, hacia la obtención de un software de baja calidad [21].

\section{Problema Abierto}

El problema abierto que se identifica en la presente sección, consiste en la necesidad de estructurar y categorizar la masa de información proveniente del proceso de educción a los efectos de facilitar la comprensión del problema manifestado por el usuario [3], [10], [22]. En otros términos, conceptualizar los requisitos.

La insuficiencia en el tratamiento de la complejidad contenida en el discurso del usuario en la literatura correspondiente, y la necesidad de cubrirla, ha sido resaltada por diversos autores: [23], [24], [12], [25], [26], [10], [27], [15], [28], [29] entre otros. Estos autores mencionan las dificultades para la construcción de los modelos conceptuales a partir de la información recogida en el proceso de educción y plasmada en el discurso de usuario. Asimismo cabe resaltar, que dichas dificultades dotan al proceso de Análisis de un grado tal de inmadurez que hace que sea difícil llevar a cabo en forma efectiva esta actividad, al mismo tiempo que dificulta la adopción de este enfoque en las organizaciones.

Por consiguiente y en virtud de todo lo expuesto, el problema abierto que se aborda en este artículo, consiste en la existencia de una "brecha conceptual", lo que se denomina un "gap" [23], [3], [30] en la transición de un proceso (Educción de Requisitos) a otro proceso (Modelado Conceptual). Este concepto se ilustra en la figura 2 :

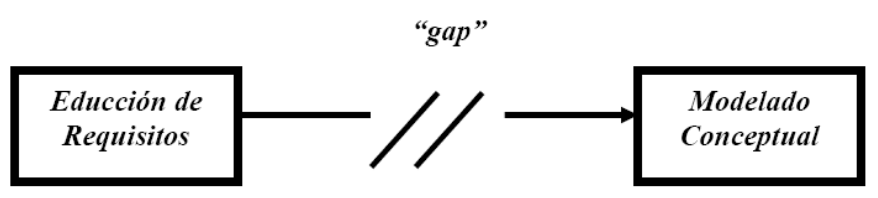

Fig. 2. Representación del "gap" entre los procesos de Educción de Requisitos y Modelado Conceptual

A causa de lo expuesto, se manifiesta la necesidad de conceptualizar los requisitos manifestados por el usuario en su discurso antes de pasar a la construcción de los modelos conceptuales, con el objeto de reducir la complejidad mencionada y favorecer la comprensión del problema planteado por el usuario, contribuyendo así a la obtención de Modelos Conceptuales de mayor calidad [19], [4].

Asimismo, es importante señalar la muy escasa cantidad de trabajos existentes en la literatura científica sobre la elaboración de representaciones intermedias de los caudales de información obtenidos por el IR en el proceso de educción. En otras palabras, trabajos que estén orientados a la búsqueda de reducción de la complejidad de la realidad y su problemática expresada por el usuario en su discurso.

El Modelo de Proceso de Conceptualización de Requisitos que se presenta en la Sección III, correspondiente a la Solución del problema identificado, pretende realizar un aporte en este sentido. Con el soporte conceptual de tópicos pertenecientes a otras disciplinas, tales como el Análisis de Protocolo proveniente del campo de la Ingeniería del Conocimiento; y las Técnicas Cognitivas propias del campo de las Teoría Educativas, se analiza en detalle el Discurso del Usuario a los fines de estructurar y caracterizar el cuerpo de información presente en el mismo.

\section{E. Sumario de Investigación}

De lo expuesto precedentemente surgen las siguientes preguntas de investigación:

Pregunta 1: ¿Se puede plantear distinciones en el discurso del usuario que permitan diferenciar subdominios de análisis que minimicen la brecha conceptual entre 
la educción de requisitos y el modelado conceptual? En caso afirmativo: ¿Cuales?

Pregunta 2: ¿De existir tales distinciones, se puede plantear un proceso que permita transformar el discurso del usuario en un conjunto de formalismos que lo sistematicen y lo documenten? De ser posible: ¿Cuáles son las fases de dicho proceso, las tareas vinculadas a cada fase y las técnicas asociadas a cada tarea?

Se proponen soluciones a los interrogantes planteados en la próxima sección.

\section{SOLUCIÓN}

En esta sección se presenta: un Modelo de proceso de conceptualización de Requisitos, del cual se abordan las cuestiones generales de mayor relevancia, se presenta la propuesta de dicho modelo, la que se describe a partir de su estructura general, los escenarios de usuario, y el enfoque cognitivista en la construcción de los mismos. Luego se explican en detalle las dos fases que componen el modelo (fase de Análisis Orientado al Problema y fase de Análisis Orientado al Producto), junto con las tareas que deben realizarse para llevar a cabo estas fases y los productos que se obtienen con la implementación de las mencionadas tareas. Se introducen las técnicas asociadas a las tareas del modelo de proceso. En primer término se presentan las técnicas utilizadas en la fase de Análisis Orientado al Problema como: la Técnica de Segmentación del Discurso de Usuario (TS - DU), las Técnicas Cognitivas de Identificación de Conocimientos Factuales, Procedurales, Contextuales y de Asociación (TCI CFPCA) y la Técnica de Construcción del Diagrama de Espacio Problema de Escenarios de Usuario (TCD - EPEU). En segundo término se presentan las técnicas utilizadas en la fase de Análisis Orientado al Producto como: la Técnica de Construcción del Diagrama de Escenarios de Usuario (TCD EU), la Técnica de Refinamiento del Diagrama de Escenarios de Usuario (TRD - EU) y la Técnica de Construcción del Diagrama del Mapa Unificado de Escenarios de Usuario Refinados (TCD - MUEUR).

\section{A. Modelo de proceso de conceptualización de requisitos}

En esta sección se presenta una propuesta de modelo de proceso de conceptualización de Requisitos estructurada en tres partes: generalidades, propuesta del modelo de proceso de conceptualización de requisitos y fases, tareas y productos.

\section{1) Generalización}

En función del análisis realizado en la sección correspondiente a la Descripción del Problema, se considera de interés citar nuevamente el problema abierto que se aborda en este artículo, recordando que el mismo se focaliza en la "brecha conceptual" o "gap" presente en la transición del proceso de educción de requisitos de usuario al proceso de modelado conceptual. Esta brecha conceptual dificulta la comprensión del problema manifestado por el usuario $\mathrm{y}$, en consecuencia, constituye un escollo importante para la comprensión del problema manifestado por el usuario por parte del Ingeniero de Requisitos (de ahora en más IR) [23], [3], [30]. La figura 2 ilustra este concepto.

La solución que se propone en este trabajo consiste en la inserción de una actividad de "Conceptualización de Requisitos", la cuál tiene como finalidad actuar a modo de puente o enlace ("link") entre las actividades de educción de requisitos y modelado conceptual, facilitando de esta manera la comprensión del problema manifestado por el usuario y, en consecuencia, la obtención de Modelos Conceptuales de mayor calidad [4], [19], [15], [28], [29]. La ilustración de esta idea se puede visualizar en la figura 3 y en la cual se puede observar la ausencia del "gap", el cual se sustituye por la actividad de "Conceptualización de Requisitos".

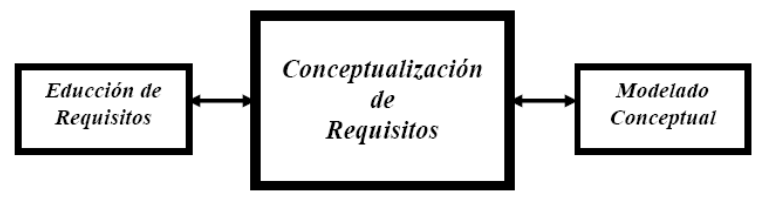

Fig. 3 Inserción de la actividad de "Conceptualización de Requisitos" entre las actividades de Educción de Requisitos y Modelado Conceptual

La idea de "conceptualizar" los requisitos de usuario por medio la actividad de "Conceptualización de Requisitos" antes de pasar a la confección de los modelos conceptuales, intenta cubrir esta "brecha conceptual" o "gap" [23], [3], [30] existente en la transición de un proceso (Educción de Requisitos) a otro proceso (Modelado Conceptual), actuando a modo de puente o enlace ("link") entre dichos procesos. De este modo, se busca establecer una adecuada conexión entre los mismos a partir de la inserción de la actividad de Conceptualización de Requisitos. Este artículo propone el Proceso de Conceptualización de Requisitos como instrumentación de dicha actividad.

A partir de la implementación de esta actividad de conceptualización de requisitos es posible la consecución de un conjunto de representaciones gráficas denominadas Representaciones Intermedias de los Requisitos de Usuario (RIRU), a partir de las cuales es posible "caracterizar" la información contenida en el discurso del usuario (por lo general en formato de "lenguaje natural" y es así como se la supone presentada en este trabajo), a los efectos de que sea mas sencillo su procesamiento para la construcción de los modelos conceptuales. Estas representaciones intermedias estarán conformadas, fundamentalmente, por un conjunto de representaciones gráficas: los Escenarios de Usuario Refinados (EUR), los cuales enlazados en forma adecuada a través del Mapa Unificado de Escenarios de Usuario Refinados MUEUR) permiten caracterizar el discurso del usuario en una forma alternativa al lenguaje natural clásico.

2) Propuesta del Modelo de Proceso de Conceptualización de Requisitos

En esta sección se describe la estructura general del proceso de conceptualización de requisitos y su modo de funcionamiento, un análisis de los escenarios de usuario como soporte fundamental del proceso de conceptualización de requisitos y un abordaje del enfoque cognitivista para la construcción de estos escenarios de usuario.

\section{a) Estructura General del Proceso de Conceptualización} de Requisitos

La actividad de conceptualización de requisitos se lleva a cabo por medio de un proceso dual que se denomina Proceso de Conceptualización de Requisitos, el cual está estructurado en dos fases, a saber:

i. Una primera fase de Análisis Orientado al Problema, cuyo objetivo se focaliza en la comprensión del problema 
planteado por el usuario en el dominio en el cual este tiene lugar.

ii. Una segunda fase de Análisis Orientado al Producto, cuyo objetivo consiste en la obtención de las funcionalidades que el usuario pretende obtener del producto software a desarrollar, teniendo en cuenta la vinculación de estas funcionalidades con la realidad manifestada por el usuario en su discurso.

Este proceso toma como punto de partida el Discurso de Usuario en Lenguaje Natural (DULN) y proporciona como salida el conjunto de Representaciones Intermedias de los Requisitos de Usuario (RIRU). La figura 4 ilustra este concepto:

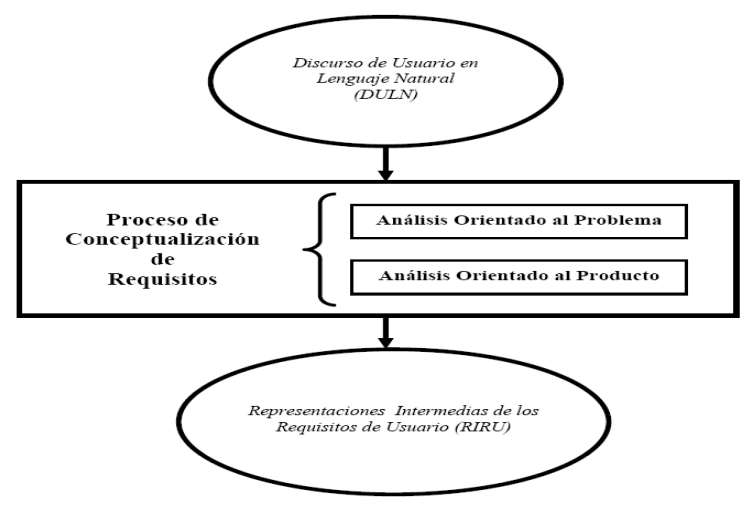

Fig. 4 Estructura General del "Proceso de Conceptualización de Requisitos" con sus dos fases y los elementos de entrada y salida

El soporte principal del Proceso de Conceptualización de Requisitos está compuesto por sus dos Fases, donde cada una de ellas está conformada por tres Tareas, y un conjunto de productos que pueden actuar como elemento de entrada y/o de salida de una determinada tarea. En otros términos, cada tarea precisa de ciertos productos para su realización, los cuales se procesan para proporcionar los correspondientes productos de salida. En la figura 4 se ilustra el modo de funcionamiento del proceso de conceptualización en base a la interdependencia conceptual existente entre la Fases, las Tareas y los Productos. En tal sentido, dicha figura muestra el flujo que siguen estos productos abasteciendo a determinadas tareas para su realización y/o ser procesados para constituirse en salida de las mismas.

En figura 5 se puede observar que en la primera fase de Análisis Orientado al Problema la primera tarea que se lleva a cabo es la de Segmentación del Discurso de Usuario (SDU), la cual necesita del Discurso de Usuario (DU) como producto de entrada y proporciona como producto de salida los correspondientes Segmentos de Texto (ST). Estos ST constituyen a su vez el producto de entrada para la realización de la tarea de Análisis Cognitivo de los Segmentos de Texto (ACST), la cual arroja como producto de salida los Tipos de Conocimiento (TC) embebidos en estos segmentos. A su vez, estos Tipos de Conocimiento (TC) junto con los Segmentos de Texto (ST) conforman el conjunto de productos de entrada necesarios para llevar a cabo la tarea de Construcción del Espacio Problema en Escenarios de Usuario (CEPEU), a partir de la cual se obtiene como producto de salida los correspondientes Espacio Problema en Escenarios de Usuario (EPEU).

Luego se comienza con el desarrollo de la fase de Análisis Orientado al Producto donde la primera tarea que se realiza es la de Construcción de Escenarios de Usuario (CEU), la cual necesita como productos de entrada a los Segmentos de Texto con Tipo de Conocimiento de Asociación (STTCA) y los Espacio Problema en Escenarios de Usuario (EPEU), los cuales se procesan en el desarrollo de esta tarea y se obtienen los respectivos Escenarios de Usuario (EU).

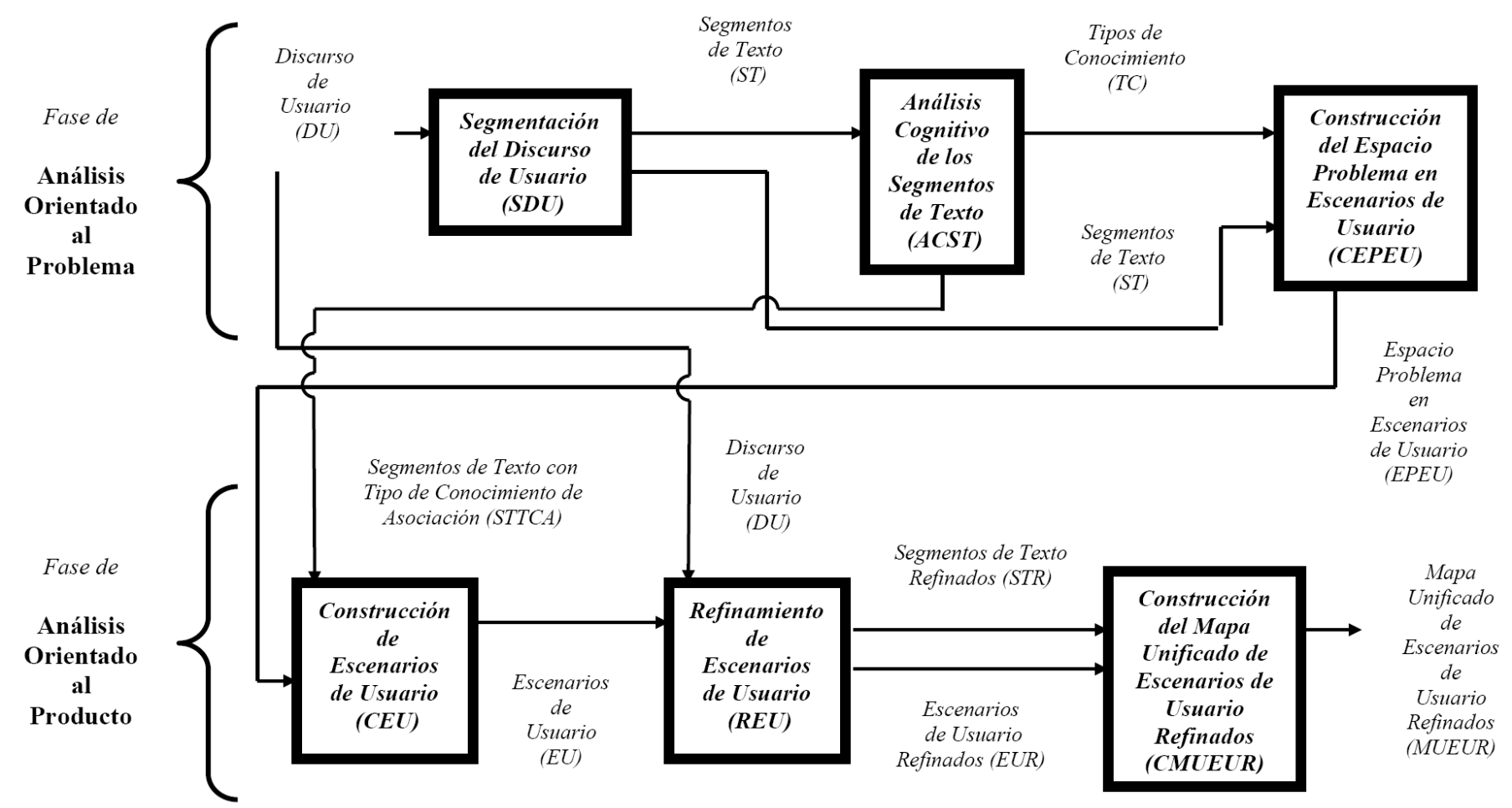

Fig. 5 Interdependencia conceptual entre las Fases, Tareas y Productos 
Estos Escenarios de Usuario (EU) junto con el Discurso de Usuario (DU) constituyen los productos de entrada para la realización de la tarea de Refinamiento de Escenarios de Usuario (REU), la cual proporciona como producto de salida los correspondientes Escenarios de Usuario Refinados (EUR). Finalmente, con los Escenarios de Usuario Refinados (EUR) y los Segmentos de Texto Refinados (STR) como productos de entrada, se realiza la tarea de Construcción del Mapa Unificado de Escenarios de Usuario Refinados (CMUEUR) y se obtiene el Mapa Unificado de Escenarios de Usuario Refinados (MUEUR).

\section{b) Escenario de Usuario}

Esta sección se focaliza en el análisis de escenarios de usuario, cuyo concepto se presenta en la sección “ $i$ ”, luego se presentan aquellas propiedades y elementos propios de un escenario de usuario que contribuyen a su caracterización (sección ii), y finalmente se proporciona un ejemplo sencillo correspondiente a un escenario de usuario (sección iii).

El concepto de Escenario de Usuario (EU) junto con sus elementos y propiedades, constituyen el soporte conceptual sobre el cual se basa la presente propuesta del proceso de conceptualización de requisitos.

\section{i. $\quad$ Concepto de Escenario de Usuario}

En el contexto del presente artículo, es sustancial precisar el concepto de Escenario de Usuario (EU) a los efectos de su caracterización y su posterior construcción. En este sentido, se proporciona la siguiente definición:

"Descripción textual o gráfica de una situación determinada que tiene lugar en el ámbito de aplicación del producto software a desarrollar y que guarda una cierta relación con la realidad (y el problema a resolver) manifestada por el usuario en su discurso"

En base a esta definición, la cual está inspirada en diversos autores que han abordado con anterioridad el estudio de esta técnica como por ejemplo [31], [32], [33], [34], [35], [36], es posible establecer pautas conceptuales que permitan una adecuada caracterización del concepto de escenario de usuario, facilitando de esta manera su proceso de construcción. La correcta confección de los escenarios de usuario le permite al IR elaborar otras herramientas de representación gráfica de los requisitos de usuario, tal como el Mapa de Escenarios de Usuario (MEU), que contribuyen a mejorar la comprensión del problema manifestado por el usuario.

\section{ii. Caracterización de un Escenario de Usuario}

A continuación se presentan las propiedades y los elementos de un escenario de usuario que contribuyen a su caracterización:

- El escenario de usuario constituye una representación, tal como un cuadro o una escena, cuya descripción intenta capturar una situación determinada de la realidad descripta por el usuario en su discurso [37].

- El escenario de usuario, como elemento descriptivo de la realidad, tiene lugar en un contexto concreto y bien definido, lo cual contribuye a que todos los elementos que conforman el escenario adquieran verdadero sentido e identidad [38].

- En el presente trabajo, el escenario de usuario se representa por medio de un esquema bidimensional (en forma de gráfico rectangular), el cual se caracteriza por contener los siguientes elementos:
1. El escenario de usuario contiene Actores (conceptos, objetos o personas del mundo real), cuya presencia y actividad son relevantes para la situación de la realidad capturada por el escenario.

2. Dentro de un escenario de usuario los actores poseen una identidad definida caracterizada por Atributos que asumen valores determinados, los cuales determinan en que estado se encuentra un actor en ese escenario de usuario (instanciación del actor).

3. Dentro de un escenario de usuario los actores pueden vincularse entre sí mediante Relaciones, las cuales ponen de manifiesto la forma en que los actores se vinculan en el marco de la situación de la realidad contenida en el escenario.

4. Dentro de un escenario de usuario los actores pueden llevar a cabo Acciones, a través de las cuales es posible determinar como el comportamiento de un actor en el escenario impacta sobre el mismo modificando su propio estado, es decir alterando el valor de alguno de los atributos del propio actor que las realiza.

Asimismo, estas acciones pueden estar caracterizadas por medio de atributos que contribuyan a describir aspectos particulares de las mismas; así como también, es posible que sea menester que deban cumplirse ciertas condiciones para que estas acciones puedan llevarse a cabo.

5. Dentro de un escenario de usuario los actores pueden llevar a cabo Interacciones, a través de las cuales es posible determinar como el comportamiento de un actor en el escenario impacta sobre otro actor modificando el estado de este último; es decir, alterando el valor de alguno de sus atributos y pudiendo ser que también se produzca un condicionamiento en el comportamiento del actor o se active en este una determinada conducta como consecuencia de la interacción. Asimismo, estas interacciones pueden estar caracterizadas por medio de atributos que contribuyan a describir aspectos particulares de las mismas; así como también, es posible que sea menester que deban cumplirse ciertas condiciones para que estas interacciones puedan llevarse a cabo.

6. A los efectos de que el formalismo de escenarios refleje la relación que debe existir entre la realidad manifestada por el usuario en su discurso y la solución software que debe resolver el problema (embebido en esta realidad); el escenario de usuario debe contener las Funcionalidades requeridas por el usuario vinculadas con los elementos de la realidad (actores, atributos, acciones, interacciones) sobre los cuales se aplican estas funcionalidades.

- En base a la caracterización realizada, se asume que la estructura de un escenario de usuario está conformada por dos bloques interconectados, identificados como Espacio Problema y Espacio - Producto; siendo que el primer bloque contiene aquellos elementos descriptivos de la realidad manifestada por el usuario en su discurso (actores, atributos, valores de atributos, relaciones, acciones e interacciones), mientras que el segundo bloque contiene las distintas funcionalidades (calcular montos, almacenar datos 
relevantes, entre otros) que el producto software debe realizar sobre la realidad modelada en el espacio - problema, y conforme a los requerimientos de usuario.

- La conexión de ambos bloques en el escenario de usuario, se representa por medio de una flecha dirigida desde el elemento del espacio - problema sobre el cual se aplica una determinada funcionalidad, hasta la correspondiente funcionalidad.

A modo de síntesis, se puede inferir que la realidad representada en el escenario de usuario a través de todos los elementos que lo conforman contextualiza el problema que se presenta, y contiene toda aquella información relevante para el entendimiento de la realidad y el problema manifestado por el usuario.

A continuación, se ilustra en figura 6 el esquema gráfico general que presenta un escenario de usuario con todos los elementos descriptos. En este sentido, se entiende que para un determinado escenario de usuario no todos los elementos mencionados en la caracterización deben estar presentes; podría ser por caso, que no se registren acciones de los actores que originen modificación de su propio estado cambiando algún valor de alguno de sus atributos o que la interacción entre dos actores no posea atributos. En la parte inferior del esquema de representación de figura 6 se coloca una etiqueta de denominación del escenario de usuario con una numeración correlativa en número romano $(\mathrm{EU}-\mathrm{I})$.

Tanto en las acciones como en las interacciones, se representa en el recuadro el atributo correspondiente junto con su valor, además de alguna condición que pudiera tener que cumplirse para que la acción o interacción tenga lugar. Por ejemplo, si fuese el caso de una interacción como Comunicar, en el recuadro se tendría Velocidad - Rápida (atributo con su valor) y Radio Encendida (condición para que se realice la interacción).

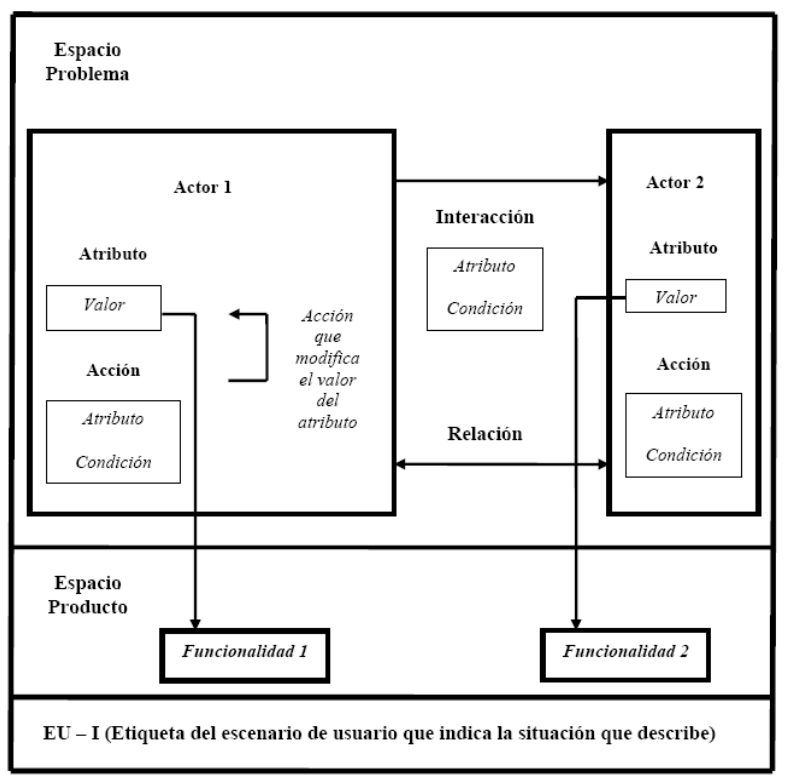

Fig. 6 Representación gráfica de un "Escenario de Usuario" con sus elementos constitutivos

\section{iii. Ejemplo de Escenario de Usuario}

A los efectos de esclarecer la caracterización realizada en el apartado anterior, se analiza el siguiente caso correspondiente a un sistema de gestión de mantenimiento de aeronaves, asumiendo que los elementos que conforman el escenario de usuario ya fueron obtenidos a partir del análisis del discurso de usuario.

Se supone la siguiente caracterización del escenario que se analiza:

El presente escenario captura una situación que representa una porción o segmento de un discurso de usuario, quien podría estar representado por el Jefe de Mantenimiento de Aeronaves, que tiene lugar en un determinado contexto (sector de mantenimiento de un aeropuerto) y que muestra como es el mecanismo de abastecimiento de combustible de una aeronave.

El proceso de construcción del escenario de usuario a partir del análisis de su discurso será explicado posteriormente en esta sección como parte de la metodología que se propone. No obstante, y supuesto realizado este análisis, se asume que a partir del mismo la idea central del segmento de discurso de usuario que se refiere al proceso de abastecimiento de una aeronave en el sector de mantenimiento de un aeropuerto, refleja la siguiente realidad precisada por el usuario y recogida por el IR:

"La aeronave debe poseer una identificación, conocerse su ubicación, si tiene realizado el mantenimiento mecánico y si sus motores están encendidos; asimismo, dado que hay más de una torre de control que controla el movimiento de los aviones, es preciso saber cuál de ellas (cada una posee un número que la identifica) es la que autoriza el abastecimiento. Los demás detalles de esta parte del discurso de usuario, especifican relaciones entre los actores identificados, acciones, interacciones con sus atributos y condiciones. Asimismo, también se relevaron funcionalidades que debe llevar a cabo la aplicación software; tales como llevar un registro de los autorizaciones de abastecimiento que han sido aceptadas por la torre de control en un día determinado y la cantidad total de los mantenimientos mecánicos que han sido realizados en todas las aeronaves que operaron en el sector de mantenimiento en ese mismo día"

En tal sentido, a continuación se detallan los diferentes elementos que conforman el correspondiente escenario de usuario junto con las funcionalidades que se aplican sobre estos:

1. Actores relevantes para la situación de la realidad capturada por el escenario:

- Aeronave

- $\quad$ Torre de Control

2. Atributos relevantes de cada uno de estos actores para el desarrollo de su actuación en el escenario y posibles valores que pueden tomar estos atributos, determinando así el estado en que se encuentran cada uno de estos actores en el escenario, es decir, su instanciación:

- Atributos del actor Aeronave:

Identificación: posee un valor, por ejemplo $341 \mathrm{H} 2048$

Ubicación: toma un valor para un momento dado, por ejemplo el Hangar $N^{\circ} 1$

Mantenimiento Mecánico: toma un valor para un momento dado, por ejemplo el Realizado o No Realizado

Estado de Motores: toma un valor para un momento dado, por ejemplo Encendidos o No Encendidos 
- Atributos del actor Torre de Control:

Número: posee un valor, por ejemplo Torre $N^{\circ} 2$

Autorización de Abastecimiento: puede tomar el valor Afirmativa o Negativa

3. Relaciones que ponen de manifiesto la forma en que los actores se vinculan en el marco de la situación de la realidad contenida en el escenario de usuario:

- Relación entre el actor Aeronave y Torre de Control:

Controla: esta relación estipula que las torres de control supervisan el movimiento de los aviones para la recarga de combustible

4. Acciones a través de las cuales un actor modifica su propio estado alterando el valor de alguno de sus atributos; se señalan atributos y condiciones que se deben cumplir para que estas acciones puedan llevarse a cabo:

- Acción que lleva a cabo el actor Aeronave:

Mover: modifica el valor del atributo ubicación del actor aeronave, con lo cual cambia su estado; esta acción posee el atributo Velocidad que adopta un valor determinado (por ejemplo $20 \mathrm{~km} / \mathrm{h}$ ) y la condición que debe cumplirse para que la acción tenga lugar es que la aeronave tenga los Motores Encendidos.

5. Interacciones a través de las cuales un actor modifica el estado de otro actor con el cual interactúa alterando el valor de alguno de sus atributos; se señalan atributos y condiciones que se deben cumplir para que estas interacciones puedan llevarse a cabo:

- Interacción que tiene lugar entre los actores Aeronave y Torre de Control:

Pedido de Autorización: por medio de esta interacción el actor Aeronave solicita autorización para abastecerse de combustible al actor Torre de Control.

Decisión sobre Autorización: por medio de esta interacción el actor Torre de Control toma una decisión (afirmativa o negativa) sobre el pedido de autorización y se lo informa al actor Aeronave. Ambas interacciones poseen el atributo referido al Tipo de Comunicación que puede ser Simplex, Duplex o Full - Duplex; a su vez, la condición que debe cumplirse para que tengan lugar estas interacciones es que la aeronave tenga el Mantenimiento Mecánico Realizado.

6. Funcionalidades requeridas por el usuario vinculadas con los elementos de la realidad (atributos, acciones, interacciones, etc) sobre los cuales se aplican estas funcionalidades:

- Funcionalidad que se aplica sobre el atributo Mantenimiento Mecánico del actor Aeronave:

Cálculo del total de los mantenimientos mecánicos realizados en todas las aeronaves en un día: por medio de esta funcionalidad el usuario desea conocer la totalidad de los mantenimientos mecánicos realizados por el sector sobre todas las aeronaves en un día determinado.
- Funcionalidad que se aplica sobre el atributo Autorización de Abastecimiento del actor Torre de Control:

Registro de las autorizaciones de abastecimiento aceptadas por la torre de control en un día: por medio de esta funcionalidad el usuario desea tener un registro de aquellas autorizaciones de abastecimiento que fueron aceptadas por la torre de control en un día.

Tal como se puede apreciar en el escenario correspondiente a la figura 7, el mismo representa una instantánea de la realidad reflejada en una parte del discurso de usuario, junto con las prestaciones o funcionalidades que el usuario pretende obtener del producto software a construir. Esto es así en virtud de que los actores representados están instanciados con valores determinados para sus correspondientes atributos.

A continuación se ilustra en figura 7 el esquema gráfico correspondiente a este escenario de usuario con su Espacio Problema y Espacio - Producto y todos los elementos que fueron identificados por el IR a partir del análisis del discurso de usuario.

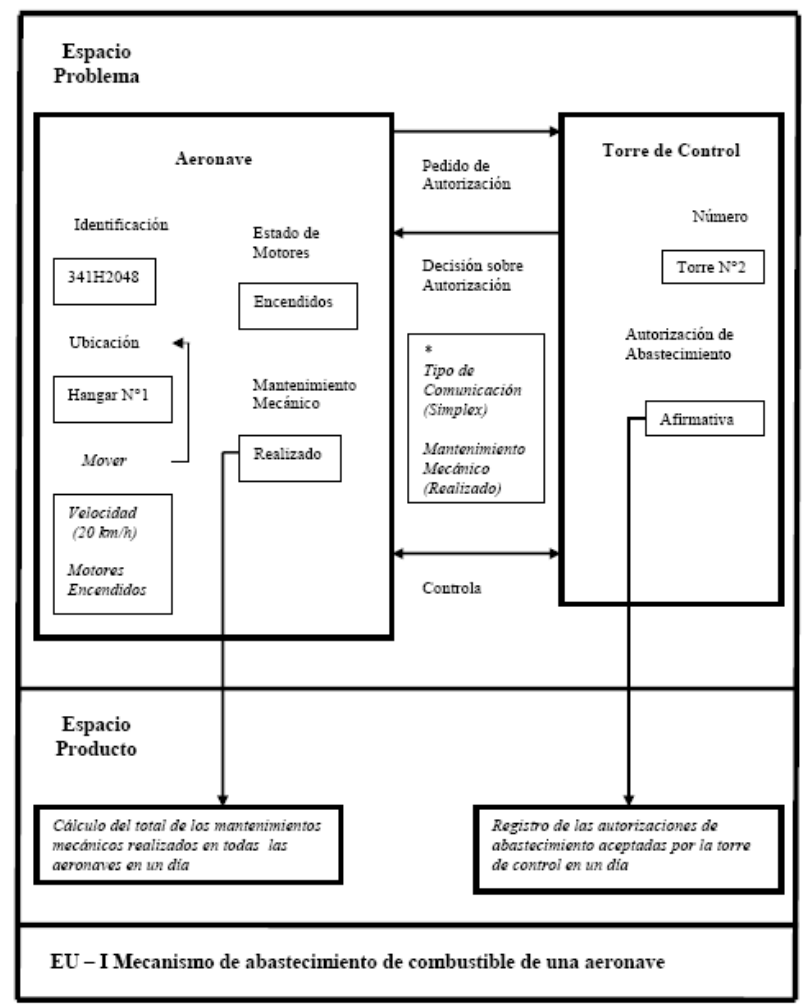

Fig. 7 Representación gráfica del "Escenario de Usuario" correspondiente al mecanismo de abastecimiento de combustible de una aeronave en el sector de mantenimiento de un aeropuerto

* Significa que el atributo y la condición son válidas para ambas interacciones de Pedido de Autorización y Decisión sobre Autorización.

\section{c) Enfoque Cognitivista en la Construcción de Escenario} de Usuario

En esta sección se abordan los aspectos generales del Enfoque Cognitivista, el cual constituye el soporte fundamental para la construcción de los Escenarios de Usuario (EU). En este sentido y para una mejor comprensión acerca del uso de este enfoque en la construcción de los EU, es 
importante destacar que a partir de la información proporcionada por el usuario en su discurso, el IR debe capturar una realidad que incluye un problema que se debe resolver por medio de un producto software. Esta realidad constituye un elemento "intangible" y, por lo general, presenta un importante grado de complejidad; lo cual hace que no resulte sencilla su captura $y$, por consiguiente, la tarea de modelarla.

Asimismo, es importante destacar que el discurso del usuario constituye una declaración textual de cómo este usuario vivencia (o vivenció) esta realidad junto con el problema a resolver. Esta vivencia le permite al usuario crear en su mente un modelo de la realidad y el problema el cual constituye un importante capital en lo que se refiere a la experiencia de dicha realidad que el IR no posee, lo que origina dificultades en la comprensión de esta realidad y su conexión con el problema a resolver [23], [3].

Tal como se ha especificado la construcción de este "Modelo Mental" o "Estructura Cognitiva" constituye un proceso mental indexado por vivencias y experiencias que son de carácter netamente personal y que tienen lugar en determinados contextos [39], [40]. Conforme a investigaciones llevadas a cabo en Psicología Cognitiva por John Black, este modelo mental contiene de forma integrada diferentes clases de conocimiento [41] [42] tales como: conocimiento factual, conocimiento procedural, conocimiento contextual y conocimiento de asociación.

Cabe consignar que estos tipos de conocimiento se encuentran presentes en el discurso del usuario y su identificación le permite al IR obtener los distintos elementos que componen los escenarios de usuario (actores, atributos, relaciones, acciones, interacciones y funcionalidades), para luego proceder a la construcción de los mismos. En la sección correspondiente a la presentación de las diferentes técnicas que permiten implementar las tareas que componen cada una de las fases del proceso de conceptualización, se explica en detalle la forma de obtener estos elementos y de construir estos escenarios de usuario.

\section{3) Fases, Tareas y Productos}

En esta sección se presenta un breve sumario acerca de los diferentes elementos que conforman el soporte del Proceso de Conceptualización de Requisitos, poniendo énfasis en la localización de cada uno de ellos dentro de la estructura del proceso y de como intervienen para que el mismo pueda llevarse a cabo. Tal como se especificó en la sección de Estructura General del Proceso de Conceptualización de Requisitos, este se desarrolla por medio de la implementación de dos fases fundamentales: la fase de Análisis Orientado al Problema y la fase de Análisis Orientado al Producto. Para la realización de cada una de estas fases es necesario llevar a cabo una serie de tareas, las cuales tienen como función el procesamiento de ciertos productos de entrada para obtener los correspondientes productos de salida. Este procesamiento de los productos de entrada se efectúa a través de una determinada Técnica de Transformación especialmente diseñada para cada tarea. En otras palabras, existe una relación biunívoca entre cada una de las tareas que conforman cada fase del proceso y su correspondiente técnica de transformación asociada.

En función de lo expuesto, para la fase de Análisis Orientado al Problema se tienen las siguientes relaciones entre las tareas y las técnicas que se deben aplicar: para el desarrollo de la tarea Segmentación del Discurso de Usuario (SDU) se aplica la Técnica de Segmentación del Discurso de Usuario (TS - DU), para la implementación de la tarea Análisis Cognitivo de los Segmentos de Texto (ACST) se aplican las Técnicas Cognitivas de Identificación de Conocimientos Factuales, Procedurales, Contextuales y de Asociación (TCI CFPCA) y para realizar la tarea Construcción del Espacio Problema de Escenarios de Usuario (CEPEU) se aplica la Técnica de Construcción del Diagrama de Espacio Problema de Escenarios de Usuario (TCD - EPEU).

En lo que respecta a la fase de Análisis Orientado al Producto se tienen las siguientes relaciones entre las tareas y las técnicas que se deben aplicar: para el desarrollo de la tarea Construcción de Escenarios de Usuario (CEU) se aplica la Técnica de Construcción del Diagrama de Escenarios de Usuario (TCD - EU), para la implementación de la tarea Refinamiento de Escenarios de Usuario (REU) se aplica la Técnica de Refinamiento del Diagrama de Escenarios de Usuario (TRD - EU) y para realizar la tarea Construcción del Mapa Unificado de Escenarios de Usuario Refinados (CMUEUR) se aplica la Técnica de Construcción del Diagrama del Mapa Unificado de Escenarios de Usuario Refinados (TCD - MUEUR).

En figura 8 se ilustra la relación entre las fases, tareas y productos (con su correspondiente formato de representación) que componen el proceso de conceptualización de requisitos:

\section{Técnicas asociadas a las tareas del modelo de proceso}

En esta sección se proponen un conjunto de técnicas que le permiten al IR implementar las correspondientes tareas que conforman el Proceso de Conceptualización de Requisitos. En este sentido, se proponen técnicas que se utilizan para el desarrollo de las fases de Análisis Orientado al Problema y Análisis Orientado al Producto.

1) Técnicas Utilizadas en la Fase de Análisis Orientado al Problema

En esta sección se describen las técnicas utilizadas para el desarrollo de las tareas correspondientes a la fase de Análisis Orientado al Problema: Técnica de Segmentación del Discurso de Usuario (TS - DU), Técnicas Cognitivas de Identificación de Conocimientos Factuales, Procedurales, Contextuales y de Asociación (TCI - CFPCA) y Técnica de Construcción del Diagrama de Espacio Problema de Escenarios de Usuario (TCD-EPEU).

a) Técnica de Segmentación del Discurso de Usuario $(T S-D U)$

Por medio de esta técnica se implementa la primera tarea que debe llevar a cabo el IR en la fase de Análisis Orientado al Problema, denominada Segmentación del Discurso de Usuario (SDU). Para la aplicación de la TS - DU el IR cuenta con el Discurso de Usuario (DU) en lenguaje natural como producto de entrada, y comienza por una segmentación de dicho DU "frase" por "frase" (llamadas "frases cortas"), luego procede a integrar estas frases en Segmentos de Texto (ST) que identifiquen situaciones de la realidad descripta por el usuario, y finalmente obtener los ST asociados a los diferentes Escenarios de Usuario (EU). 


\begin{tabular}{|c|c|c|c|c|c|c|}
\hline \multirow{2}{*}{ FASE } & \multirow{2}{*}{ TAREA } & \multicolumn{2}{|c|}{ PRODUCTOS DE ENTRADA } & \multirow{2}{*}{$\begin{array}{c}\text { TECNICA DE } \\
\text { TRANSFORMACION A UTILIZAR }\end{array}$} & \multicolumn{2}{|c|}{ PRODUCTOS DE SALIDA } \\
\hline & & ENTRADA & RFEPRESENTACION & & SALIDA & REPRESENTACIÓN \\
\hline \multirow{3}{*}{$\begin{array}{l}\text { Análisis } \\
\text { Orientado al } \\
\text { Problema }\end{array}$} & $\begin{array}{l}\text { Segmentación del } \\
\text { Discurso de Usuario } \\
\text { (SDU) }\end{array}$ & $\begin{array}{l}\text { - Discurso de Usuario } \\
\text { (DU) }\end{array}$ & - Texto Plano (TP) & $\begin{array}{l}\text { - Técnica de Segmentación } \\
\text { del Discurso de Usuario } \\
\text { (TS-DU) }\end{array}$ & $\begin{array}{l}\text { - Segmentos de Texto } \\
\text { (ST) }\end{array}$ & $\begin{array}{l}\text { - Tablas de } \\
\text { Segmentos de Texto } \\
\text { (T-ST) }\end{array}$ \\
\hline & $\begin{array}{l}\text { Análisis Cognitivo de } \\
\text { los Segmentos de } \\
\text { Texto (ACST) }\end{array}$ & $\begin{array}{l}\text { - Segmentos de Texto } \\
\text { (ST) }\end{array}$ & $\begin{array}{l}\text { - Tablas de Segmentos } \\
\text { de Texto (T-ST) }\end{array}$ & $\begin{array}{l}\text { - Técnicas Cognitivas de } \\
\text { Identificación de } \\
\text { Conocimientos Factuales, } \\
\text { Procedurales, } \\
\text { Contextuales y de } \\
\text { Asociación (TCl-CFPCA) }\end{array}$ & $\begin{array}{l}\text { - Tipos de } \\
\text { Conocimiento (TC) }\end{array}$ & $\begin{array}{l}\text { - Tabla de } \\
\text { Conocimientos } \\
\text { Factuales, } \\
\text { Procedurales, } \\
\text { Contextuales y de } \\
\text { Asociación } \\
\text { (T-TCl-CFPCA) }\end{array}$ \\
\hline & $\begin{array}{l}\text { Construcción del } \\
\text { Espacio Problema en } \\
\text { Escenarios de Usuario } \\
\text { (CEPEU) }\end{array}$ & $\begin{array}{l}\text { - Segmentos de Texto } \\
\text { (ST) } \\
\text { - Tipos de Conocimiento } \\
\text { (TC) }\end{array}$ & $\begin{array}{l}\text { - Tablas de Segmentos } \\
\text { de Texto (T-ST) } \\
\text { - Tabla de Conocimientos } \\
\text { Factuales, Procedurales } \\
\text { y de Asociación } \\
\text { (T-TCl-FPA) }\end{array}$ & $\begin{array}{l}\text { - Técnica de Construcción } \\
\text { del Diagrama de Espacio } \\
\text { Problema de Escenarios } \\
\text { de Usuario (TCD - EPEU) }\end{array}$ & $\begin{array}{l}\text { - Espacio Problema } \\
\text { en Escenarios de } \\
\text { Usuario (EPEU) }\end{array}$ & $\begin{array}{l}\text { - Diagrama de } \\
\text { Espacio Problema } \\
\text { en Escenarios de } \\
\text { Usuario (D-EPEU) }\end{array}$ \\
\hline \multirow{3}{*}{$\begin{array}{l}\text { Análisis } \\
\text { Orientado al } \\
\text { Producto }\end{array}$} & $\begin{array}{l}\text { Construcción de } \\
\text { Escenarios de Usuario } \\
\text { (CEU) }\end{array}$ & $\begin{array}{l}\text { - Segmentos de Texto } \\
\text { con Tipo de } \\
\text { Conocimiento de } \\
\text { Asociación (STTCA) } \\
\text { - Espacio Problema en } \\
\text { Escenarios de Usuario } \\
\text { (EPEU) }\end{array}$ & $\begin{array}{l}\text { - Tablas de Segmentos } \\
\text { de Texto con Tipo de } \\
\text { Conocimiento de } \\
\text { Asociación (T-STTCA) } \\
\text { - Diagrama de Espacio } \\
\text { Problema en Escenarios } \\
\text { de Usuario (D-EPEU) } \\
\end{array}$ & $\begin{array}{l}\text { - Técnica de Construcción } \\
\text { del Diagrama de } \\
\text { Escenarios de Usuario } \\
\text { (TCD - EU) }\end{array}$ & $\begin{array}{l}\text { - Escenarios de } \\
\text { Usuario (EU) }\end{array}$ & $\begin{array}{l}\text { - Diagrama de } \\
\text { Escenarios de } \\
\text { Usuario (D-EPEU) }\end{array}$ \\
\hline & $\begin{array}{l}\text { Refinamiento de } \\
\text { Escenarios de Usuario } \\
\text { (REU) }\end{array}$ & $\begin{array}{l}\text { - Discurso de Usuario } \\
\text { (DU) } \\
\text { - Escenarios de Usuario } \\
\text { (EU) }\end{array}$ & $\begin{array}{l}\text { - Texto Plano (TP) } \\
\text { - Diagrama de } \\
\text { Escenarios de Usuario } \\
\text { (D-EPEU) }\end{array}$ & $\begin{array}{l}\text { - Técnica de Refinamiento } \\
\text { del Diagrama de } \\
\text { Escenarios de Usuario } \\
\text { (TRD - EU) } \\
\end{array}$ & $\begin{array}{l}\text { - Escenarios de } \\
\text { Usuario Refinados } \\
\text { (EUR) }\end{array}$ & $\begin{array}{l}\text { - Diagrama de } \\
\text { Escenarios de } \\
\text { Usuario Refinados } \\
\text { (D-EUR) } \\
\end{array}$ \\
\hline & $\begin{array}{l}\text { Construcción del Mapa } \\
\text { Unificado de } \\
\text { Escenarios de Usuario } \\
\text { Refinados (CMUEUR) }\end{array}$ & $\begin{array}{l}\text { - Segmentos de Texto } \\
\text { (ST) } \\
\text { - Escenarios de Usuario } \\
\text { Refinados (EUR) }\end{array}$ & $\begin{array}{l}\text { - Tablas de Segmentos } \\
\text { de Texto (T-ST) } \\
\text { - Diagrama de } \\
\text { Escenarios de Usuario } \\
\text { Refinados (D-EUR) }\end{array}$ & $\begin{array}{l}\text { - Técnica de Construcción } \\
\text { del Diagrama del Mapa } \\
\text { Unificado de Escenarios } \\
\text { de Usuario (TCD - } \\
\text { MUEU) }\end{array}$ & $\begin{array}{l}\text { - Mapa Unificado de } \\
\text { Escenarios de } \\
\text { Usuario (MUEU) }\end{array}$ & $\begin{array}{l}\text { - Diagrama de Mapa } \\
\text { Unificado de } \\
\text { Escenarios de } \\
\text { Usuario Refinados } \\
\text { (D-MUEUR) }\end{array}$ \\
\hline
\end{tabular}

Fig. 8 Representación gráfica de las fases, tareas y productos con sus formatos de representación

D. Técnicas asociadas a las tareas del modelo de proceso

En esta sección se proponen un conjunto de técnicas que le permiten al IR implementar las correspondientes tareas que conforman el Proceso de Conceptualización de Requisitos. En este sentido, se proponen técnicas que se utilizan para el desarrollo de las fases de Análisis Orientado al Problema y Análisis Orientado al Producto.

2) Técnicas Utilizadas en la Fase de Análisis Orientado al Problema

En esta sección se describen las técnicas utilizadas para el desarrollo de las tareas correspondientes a la fase de Análisis Orientado al Problema: Técnica de Segmentación del Discurso de Usuario (TS - DU), Técnicas Cognitivas de Identificación de Conocimientos Factuales, Procedurales, Contextuales y de Asociación (TCI - CFPCA) y Técnica de Construcción del Diagrama de Espacio Problema de Escenarios de Usuario (TCD - EPEU).

\section{b) Técnica de Segmentación del Discurso de Usuario} $(T S-D U)$

Por medio de esta técnica se implementa la primera tarea que debe llevar a cabo el IR en la fase de Análisis Orientado al Problema, denominada Segmentación del Discurso de Usuario (SDU). Para la aplicación de la TS - DU el IR cuenta con el Discurso de Usuario (DU) en lenguaje natural como producto de entrada, y comienza por una segmentación de dicho DU "frase" por "frase" (llamadas "frases cortas"), luego procede a integrar estas frases en Segmentos de Texto (ST) que identifiquen situaciones de la realidad descripta por el usuario, y finalmente obtener los ST asociados a los diferentes Escenarios de Usuario (EU).

Los ST con los EU asociados constituyen el producto de salida que proporciona la TS - DU. La tabla 1 resume los pasos necesarios para la implementación de esta técnica.

\begin{tabular}{|l|}
\hline \multicolumn{1}{|c|}{ Técnica de Segmentación del Discurso de Usuario (TS - DU) } \\
\hline Entradas: Discurso de Usuario (DU) \\
Salidas: ST Asociados a los EU \\
\hline Paso 1. Segmentación del DU en "frases cortas" \\
Paso 2. Integración de las "frases cortas" en ST \\
Paso 3. Asociación de los ST a EU \\
\hline
\end{tabular}

Tabla 1. Técnica de Segmentación del Discurso de Usuario (TS - DU)

Paso 1: En este primer paso se realiza un análisis preliminar del DU a los efectos de segmentarlo en "frases cortas". La idea de segmentar el DU de esta forma proviene de la Ingeniería de Conocimiento y se basa en la técnica de Análisis de Protocolo para educir conocimiento de un experto cuando el mismo relata como resuelve un determinado problema [7], [8]; en este caso, en la fase de "Transcripción del Protocolo", el ingeniero de conocimiento segmenta y transcribe el relato del experto en base a cuestiones tales como las pausas que realiza el experto en su exposición o las distintas entonaciones a lo largo de su relato. Esta segmentación inicial permite un tratamiento más simple del DU para afrontar el paso 2 de este proceso. Las frases cortas obtenidas constituyen el subproducto de salida correspondiente a este paso. 
Paso 2: En este segundo paso se integran las "frases cortas" obtenidas en el paso 1 en ST descriptivos de una situación o episodio de la realidad. Estos ST están conformados por conjuntos de frases cortas y constituyen el subproducto de salida correspondiente a este paso.

Paso 3: En este tercer paso se asocia cada uno de los segmentos de texto obtenidos en el paso anterior a un escenario de usuario. Por consiguiente, como resultado de este proceso de asociación se obtienen los correspondientes ST asociados a los EU, los cuales constituyen el producto de salida de esta técnica.

La técnica propuesta y los subproductos que se obtienen se pueden visualizar en figura 9.

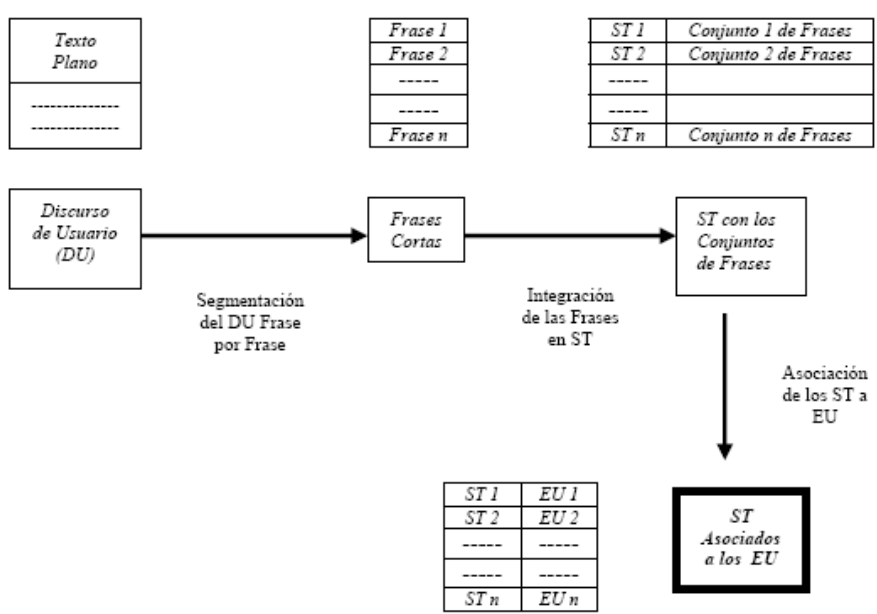

Fig. 9 Esquema y subproductos resultantes de aplicar la Técnica de Segmentación del Discurso de Usuario (TS - DU)

Nota: en cada figura y para cada uno de los productos y subproductos de entrada y salida se ilustran de manera adicional los formatos de representación.

c) Técnicas Cognitivas de Identificación de Conocimientos Factuales, Procedurales, Contextuales y de Asociación (TCI - CFPCA)

Por medio de esta técnica se implementa la segunda tarea que debe llevar a cabo el IR en la fase de Análisis Orientado al Problema, denominada Análisis Cognitivo de los Segmentos de Texto (ACST). Para la aplicación de la TCI - CFPCA el IR dispone como producto de entrada de cada uno de los ST asociados a los EU que fueron obtenidos a partir de la aplicación de la técnica anterior (TS - DU); estos segmentos se procesan con la idea de identificar en los mismos diferentes Tipos de Conocimiento (TC), los cuales se encuentran presentes en el "Modelo Mental" elaborado por el usuario a partir de un proceso mental indexado por vivencias $y$ experiencias que son de carácter netamente personal y que tienen lugar en determinados contextos [39], [40]. Conforme a investigaciones llevadas a cabo en Psicología Cognitiva por John Black, este modelo mental contiene de forma integrada diferentes TC [41], [42], entre los cuales caben citar: TC Factual, TC Procedural, TC Contextual y TC de Asociación. Para comenzar a aplicar la TCI - CFPCA el IR comienza por la identificación de TC Contextual en los ST, para luego continuar con los TC Factual, Procedural y de Asociación. Finalmente, el IR integra estos TC con los ST a los efectos de establecer que TC se corresponden con cada ST. Los ST integrados con los respectivos TC identificados (en formato de tabla) constituyen el producto de salida que proporciona la TCI - CFPCA. La tabla 2 resume los pasos y procedimientos necesarios para la implementación de esta técnica.

\begin{tabular}{|l|}
\hline $\begin{array}{c}\text { Técnica Cognitiva de Identificación de Conocimientos Factuales, } \\
\text { Procedurales, Contextuales y de Asociación (TCI - CFPCA) }\end{array}$ \\
\hline $\begin{array}{l}\text { Entradas: ST Asociados a los EU } \\
\text { Salidas: } \quad \text { TC Identificados en los ST }\end{array}$ \\
Paso 1. Identificación de TC en los ST \\
1.1. Identificación de TC Contextual en los ST \\
1.2. Identificación de TC Factual en los ST \\
1.3. Identificación de TC Procedural en los ST \\
1.4. Identificación de TC de Asociación en los ST \\
Paso 2. Integración entre los ST y TC
\end{tabular}

Tabla 2. Técnica Cognitiva de Identificación de Conocimientos Factuales, Procedurales, Contextuales y de Asociación (TCI - CFPCA)

Paso 1: En este primer paso se realiza el Análisis Cognitivo de los ST a los efectos de identificar los diferentes TC (TC Contextual, TC Factual, TC Procedural y TC de Asociación) en cada uno de los ST asociados a los EU obtenidos a partir de la aplicación de la técnica anterior. La realización de este paso se lleva a cabo por medio de cuatro procedimientos, a saber:

i. Identificación de TC Contextual en los ST: por medio de este procedimiento se realiza el Análisis Cognitivo de los ST a los efectos de identificar TC Contextual en los mismos. Este TC se caracteriza por "saber donde ocurre algo", es decir, que para la existencia de este TC el cuerpo de texto debe hacer referencia al ámbito o entorno que actúa como marco de soporte de los otros tipos de conocimiento, y por consiguiente, de la realidad descripta por el usuario. Por ejemplo, se identifica TC Contextual en frases descriptivas del tipo: "la gerencia comercial controla toda la facturación que emiten los departamentos de tesorería y finanzas antes de que sean asentadas en el libro diario". Las frases con TC Contextual constituyen el subproducto de salida correspondiente a este procedimiento.

ii. Identificación de TC Factual en los ST: por medio de este procedimiento se realiza el Análisis Cognitivo de los ST a los efectos de identificar TC Factual en los mismos. Este TC se caracteriza por "saber que algo es", es decir, que para la existencia de este TC el cuerpo de texto debe hacer referencia a frases declarativas que poseen una afirmación acerca de un hecho. Por ejemplo, se identifica TC Factual en frases tales como: "en ese caso la factura es aceptada". Las frases con TC Factual constituyen el subproducto de salida correspondiente a este procedimiento.

iii. Identificación de TC Procedural en los ST: por medio de este procedimiento se realiza el Análisis Cognitivo de los ST a los efectos de identificar TC Procedural en los mismos. Este TC se caracteriza por "saber como se hace algo", es decir, que para la existencia de este TC el cuerpo de texto debe hacer referencia a frases que sugieran un procedimiento para realizar una tarea en una cierta secuencia, o que posean una relación del tipo causa efecto. Por ejemplo, se identifica TC Procedural en frases tales como: "si la factura es aceptada entonces efectuar el pago". Las frases con TC Procedural constituyen el 
subproducto de salida correspondiente a este procedimiento.

iv. Identificación de TC de Asociación en los ST: por medio de este procedimiento se realiza el Análisis Cognitivo de los ST a los efectos de identificar TC de Asociación en los mismos. Este TC se caracteriza por "saber identificar aquellos elementos que intervienen para hacer algo", es decir, que para la existencia de este TC el cuerpo de texto debe hacer referencia a frases sugieran una cierta funcionalidad que debe realizar el sistema, con actores que intervienen para que la misma pueda llevarse a cabo. Por ejemplo, se identifica conocimiento asociado a un determinado contexto en frases del tipo: "el departamento de Recursos Humanos debe llevar un registro actualizado con los montos mensuales invertidos por departamento en concepto de capacitación de su personal". La funcionalidad o prestación a la que hace referencia esta frase del discurso "Cálculo del monto en concepto de capacitación de personal por mes y por departamento”, vincula conceptos de la realidad descripta por el usuario como los actores "Departamento de Recursos Humanos", "Departamento de Ventas", etc; que son actores necesarios para realizar esta funcionalidad. Las frases con TC de Asociación constituyen el subproducto de salida correspondiente a este procedimiento.

Los distintos TC (TC Contextual, TC Factual, TC Procedural y TC de Asociación) obtenidos constituyen el subproducto de salida correspondiente a este paso.

Paso 2: En este segundo paso se procede a integrar los ST con los TC identificados en los respectivos ST; para lo cual, se confecciona una tabla que indique los diferentes TC contenidos en cada uno de los ST. La tabla de los ST con los respectivos TC identificados constituye el producto de salida de esta técnica. La técnica propuesta y los subproductos que se obtienen se pueden visualizar en figura 10.

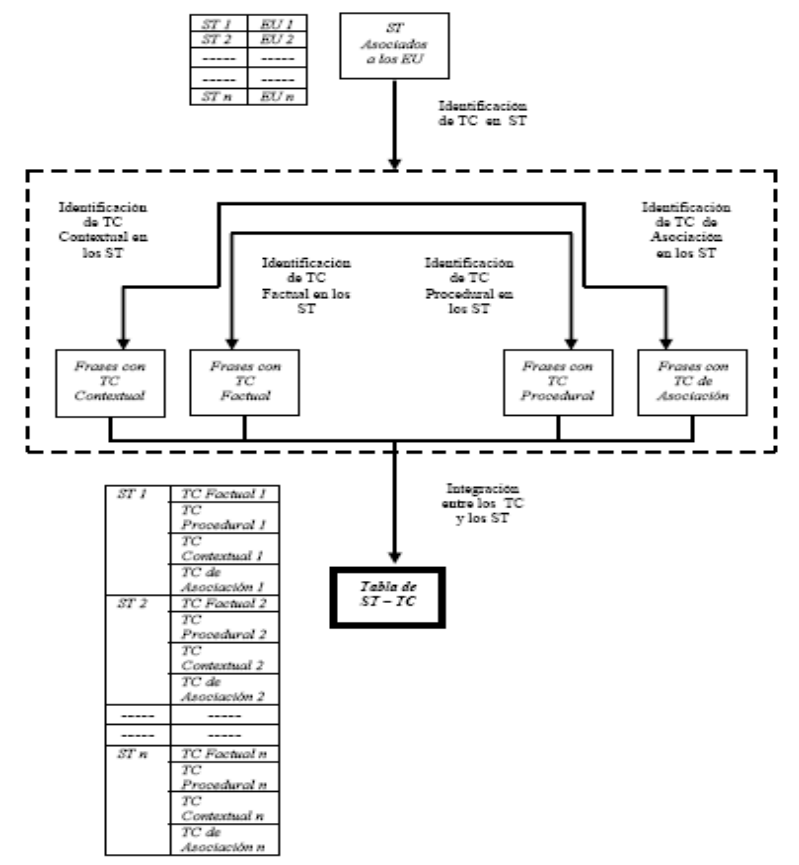

Fig. 10 Esquema y subproductos resultantes de aplicar la Técnica Cognitiva de Identificación de Conocimientos Factuales, Procedurales, Contextuales y de Asociación (TCI - CFPCA) d) Técnica de Construcción del Diagrama de Espacio Problema de Escenarios de Usuario (TCD - EPEU)

Por medio de esta técnica se implementa la tercera tarea que debe llevar a cabo el IR en la fase de Análisis Orientado al Problema, denominada Construcción del Espacio Problema en Escenarios de Usuario (CEPEU). Para la aplicación de la TCD - EPEU el IR dispone como productos de entrada de cada uno de los ST asociados a los EU obtenidos a partir de la aplicación de la técnica TS - DU, y de los TC identificados en cada uno de los ST (en formato de tabla) obtenidos a partir de la aplicación de la técnica TCI - CFPCA. Para comenzar a aplicar la TCD - EPEU el IR procede a hacer uso de los TC identificados en cada ST (dejando el TC de Asociación para la Fase de Análisis Orientado al Producto) para poder obtener los distintos elementos que componen los EPEU, los cuales son: Actores, Relaciones, Atributos, Acciones e Interacciones. A continuación, el IR procede a identificar el Marco Contextual Base $(\mathrm{MCB})$ en el que se desenvolverán los actores en el EPEU construyéndose un primer diagrama de EPEU a tal efecto. Finalmente, el IR confecciona los restantes diagramas de EPEU encargados de reflejar las distintas realidades proporcionadas por los respectivos ST.

Los diagramas correspondientes a los EPEU constituyen el producto de salida que proporciona la TCD - EPEU. La tabla 3 resume los pasos, procedimientos y subprocedimientos necesarios para la implementación de esta técnica.

Paso 1: En este primer paso el IR hace uso de los respectivos TC (Factual, Procedural y Contextual) para la identificación de los elementos que conforman los diagramas de EPEU para cada uno de los ST asociados. La realización de este paso se lleva a cabo por medio de cuatro procedimientos, a saber:

i. Uso de TC Factual, este procedimiento permite:

- identificar conceptos, que se representarán como actores en los EPEU.

- caracterizar los actores que van a conformar los respectivos EPEU, definiendo para los mismos atributos con sus respectivos valores.

- caracterizar acciones internas en los actores, definiendo para dichas acciones atributos con sus respectivos valores y condiciones para su realización.

- caracterizar interacciones entre actores, definiendo para dichas interacciones atributos con sus respectivos valores y condiciones para su realización.

- identificar las relaciones entre los actores pertenecientes a los respectivos EPEU.

ii. Uso de TC Procedural, este procedimiento permite:

- identificar acciones internas en los actores, las cuales podrán o no ser modificatorias de valores de atributo del actor.

- identificar interacciones entre actores, las cuales podrán modificar o no valores de atributo de los actores que interactúan.

iii. Uso de TC Contextual, este procedimiento permite:

- identificar el MCB o ámbito en el que tiene lugar la realidad descripta por el usuario (Departamento de Producción, Sección de Mantenimiento, etc). 
- identificar los actores y relaciones que inicialmente conforman el primer EPEU correspondiente al MCB.

\begin{tabular}{|l|}
\hline $\begin{array}{l}\text { Técnica de Construcción del Diagrama de Espacio Problema en Escenarios de Usuario (TCD - } \\
\text { EPEU) }\end{array}$ \\
Entradas: ST Asociados a los EU y Tabla de ST - TC \\
Salidas: Diagramas de EPEU \\
\hline Paso 1. Uso de los TC para la identificación de los elementos de EPEU \\
1.1. Uso de TC Factual \\
1.2. Uso de TC Procedural \\
1.3. Uso de TC Contextual \\
1.4. Elaboración de Tablas de Vinculación TC - Elementos de EPEU para cada EPEU \\
Paso 2. Construcción del Diagrama de EPEU correspondiente al MCB \\
2.1. Incorporación de Actores al Diagrama de MCB \\
2.2. Incorporación de Relaciones al Diagrama de MCB \\
Paso 3. Construcción de los restantes Diagramas de EPEU \\
Para cada uno de los Diagramas de EPEU se procede: \\
3.1. Incorporación de Actores al Diagrama \\
3.1.1. Incorporación de Atributos de actores al Diagrama \\
3.1.2. Incorporación de valores de Atributos de actores al Diagrama \\
3.2. Incorporación de Relaciones al Diagrama \\
3.3. Incorporación de Acciones al Diagrama \\
3.3.1. Incorporación de Atributos de acciones al Diagrama \\
3.3.2. Incorporación de valores de Atributos de acciones al Diagrama \\
3.3.3. Incorporación de condiciones para la realización de acciones al Diagrama \\
3.4. Incorporación de Interacciones al Diagrama \\
3.4.1. Incorporación de Atributos de interacciones al Diagrama \\
3.4.2. Incorporación de valores de Atributos de interacciones al Diagrama \\
3.4.3. Incorporación de condiciones para la realización de interacciones al Diagrama \\
\end{tabular}

Tabla 3. Técnica de Construcción del Diagrama de Espacio Problema en Escenarios de Usuario (TCD - EPEU)

iv. Uso de TC Procedural, este procedimiento permite:

- identificar acciones internas en los actores, las cuales podrán o no ser modificatorias de valores de atributo del actor.

- identificar interacciones entre actores, las cuales podrán modificar o no valores de atributo de los actores que interactúan.

v. Uso de TC Contextual, este procedimiento permite:

- identificar el MCB o ámbito en el que tiene lugar la realidad descripta por el usuario (Departamento de Producción, Sección de Mantenimiento, etc).

- identificar los actores y relaciones que inicialmente conforman el primer EPEU correspondiente al MCB.

vi. Elaboración de Tablas de Vinculación TC Elementos de EPEU para cada EPEU, este procedimiento permite sintetizar en formato de tabla toda la información correspondiente a los elementos de cada EPEU (Actores, Relaciones, Atributos, Acciones e Interacciones), obtenidos a partir de los TC contenidos en los ST.

Como resultado de la aplicación de estos procedimientos, se obtienen las respectivas Tablas de Vinculación TC Elementos de EPEU para cada EPEU, las cuales constituyen el subproducto de salida correspondiente a este paso.

Paso 2: En este segundo paso el IR procede a la construcción del diagrama de EPEU correspondiente al MCB. Para ello, el IR parte de los distintos elementos identificados en el procedimiento 1.3, junto con la Tabla de Vinculación para este EPEU. En tal sentido, y como el objetivo es establecer el marco contextual de la manera más ilustrativa posible, es que el IR representa en este diagrama los actores centrales con sus atributos de identificación (dejando la incorporación de interacciones y acciones para el próximo paso) y las relaciones entre estos, identificados en el procedimiento 1.3. Por consiguiente, para la realización de este paso se llevan a cabo los siguientes dos procedimientos:

i. Incorporación de Actores al Diagrama de EPEU del MCB: por medio de este procedimiento el IR comienza la construcción del diagrama correspondiente al MCB colocando los actores centrales identificados en el ST que contextualiza el problema.

ii. Incorporación de Relaciones al Diagrama de EPEU del MCB: por medio de este procedimiento el IR continúa la construcción del diagrama a partir de la confección de las correspondientes relaciones entre estos actores, que han sido identificadas en el ST que contextualiza el problema.

Como resultado de la aplicación de estos procedimientos, se obtienen los Actores y Relaciones que conforman el diagrama de EPEU correspondiente al MCB. Este diagrama constituye el subproducto de salida correspondiente a este paso.

Paso 3: En este tercer paso el IR procede a la construcción de los restantes diagramas de EPEU correspondientes a los ST que le continúan al del MCB. Para obtener estos diagramas, el IR parte del diagrama de EPEU del MCB, de los distintos elementos identificados en los procedimientos $1.1 \mathrm{y} 1.2$., y de las respectivas Tablas de Vinculación para cada EPEU. Por consiguiente, para la realización de este paso se llevan a cabo los siguientes cuatro procedimientos, y sus correspondientes subprocedimientos cuando correspondan, para cada uno de las correspondientes EPEU:

i. Incorporación de Actores al Diagrama de EPEU: por medio de este procedimiento el IR comienza la construcción del diagrama de EPEU correspondiente al ST de que se trate, incorporando los actores que correspondan teniendo en cuenta los ya existentes en el EPEU anterior. Se implementan los siguientes subprocedimientos:

- Incorporación de Atributos de actores al Diagrama: por medio de este subprocedimiento el IR incorpora los atributos que le permiten caracterizar a los actores.

- Incorporación de valores de Atributos de actores al Diagrama: por medio de este subprocedimiento el IR incorpora los valores correspondientes a dichos atributos.

ii. Incorporación de Relaciones al Diagrama: por medio de este procedimiento el IR continúa la construcción del diagrama de EPEU correspondiente al ST de que se trate, teniendo en cuenta las relaciones ya existentes en el EPEU anterior.

iii. Incorporación de Acciones al Diagrama: por medio de este procedimiento el IR continúa la construcción del diagrama de EPEU correspondiente al ST de que se trate, incorporando las acciones que correspondan teniendo en cuenta las ya existentes en el EPEU anterior. Se implementan los siguientes subprocedimientos:

- Incorporación de Atributos de acciones al Diagrama: por medio de este subprocedimiento el IR incorpora los atributos que le permiten caracterizar a las acciones. 
- Incorporación de valores de Atributos de acciones al Diagrama: por medio de este subprocedimiento el IR incorpora los valores correspondientes a dichos atributos.

- Incorporación de condiciones para la realización de acciones al Diagrama: por medio de este subprocedimiento el IR incorpora las condiciones correspondientes para que pueda realizarse una acción determinada.

iv. Incorporación de Interacciones al Diagrama: por medio de este procedimiento el IR continúa la construcción del diagrama de EPEU correspondiente al ST de que se trate, teniendo en cuenta las interacciones ya existentes en el EPEU anterior. Se implementan los siguientes subprocedimientos:

- Incorporación de Atributos de interacciones al Diagrama: por medio de este subprocedimiento el IR incorpora los atributos que le permiten caracterizar a las interacciones.

- Incorporación de valores de Atributos de interacciones al Diagrama: por medio de este subprocedimiento el IR incorpora los valores correspondientes a dichos atributos.

- Incorporación de condiciones para la realización de interacciones al Diagrama: por medio de este subprocedimiento el IR incorpora las condiciones correspondientes para que pueda tener lugar una interacción determinada.

La totalidad de los diagramas de EPEU con sus respectivos elementos constituyen el producto de salida de esta técnica. La técnica propuesta y los subproductos que se obtienen se pueden visualizar en figura 11.

Es muy importante destacar, que tanto en el desarrollo del Paso 2, en lo que se refiere a la construcción del Diagrama de EPEU correspondiente al Marco Contextual Base, como en el desarrollo del Paso 3, en lo que respecta a la construcción de los restantes diagramas, se estimó conveniente utilizar a modo de soporte la técnica de Análisis Estructural de Textos [5], la cual permite identificar la existencia de estructuras textuales fundamentales encargadas de transmitir conocimientos en los textos.

Las estructuras que se emplean en este trabajo son las Definiciones y Afirmaciones. Las primeras permiten detectar conceptos o actores y sus características o atributos, mientras que a partir de las segundas es posible detectar relaciones e interacciones entre conceptos.

El uso de estas estructuras le permite al IR efectuar una suerte de contraste entre los elementos de EU obtenidos con la técnica anterior (TCI - CFPCA) y los detectados por estas estructuras textuales; a la vez que son de gran utilidad para integrar gráficamente estos elementos en el "constructo" llamado EPEU.

3) Técnicas Utilizadas en la Fase de Análisis Orientado al Producto

En esta sección se describen las técnicas utilizadas para el desarrollo de las tareas correspondientes a la fase de Análisis Orientado al Producto: Técnica de Construcción del Diagrama de Escenarios de Usuario (TCD - EU), Técnica de Refinamiento del Diagrama de Escenarios de Usuario (TRD EU) y Técnica de Construcción del Diagrama del Mapa
Unificado de Escenarios de Usuario Refinados (TCD MUEUR).

a) Técnica de Construcción del Diagrama de Escenarios de Usuario (TCD - EU)

Por medio de esta técnica se implementa la primera tarea que debe llevar a cabo el IR en la fase de Análisis Orientado al Producto, denominada Construcción de Escenarios de Usuario (CEU). Para la aplicación de la TCD - EU el IR dispone como productos de entrada de aquellos ST asociados a los EU que contienen TC de Asociación, obtenidos a partir de la aplicación de las técnicas TCI - CFPCA, y de cada uno de los Diagramas de EPEU obtenidos a partir de la aplicación de la técnica TCD - EPEU.

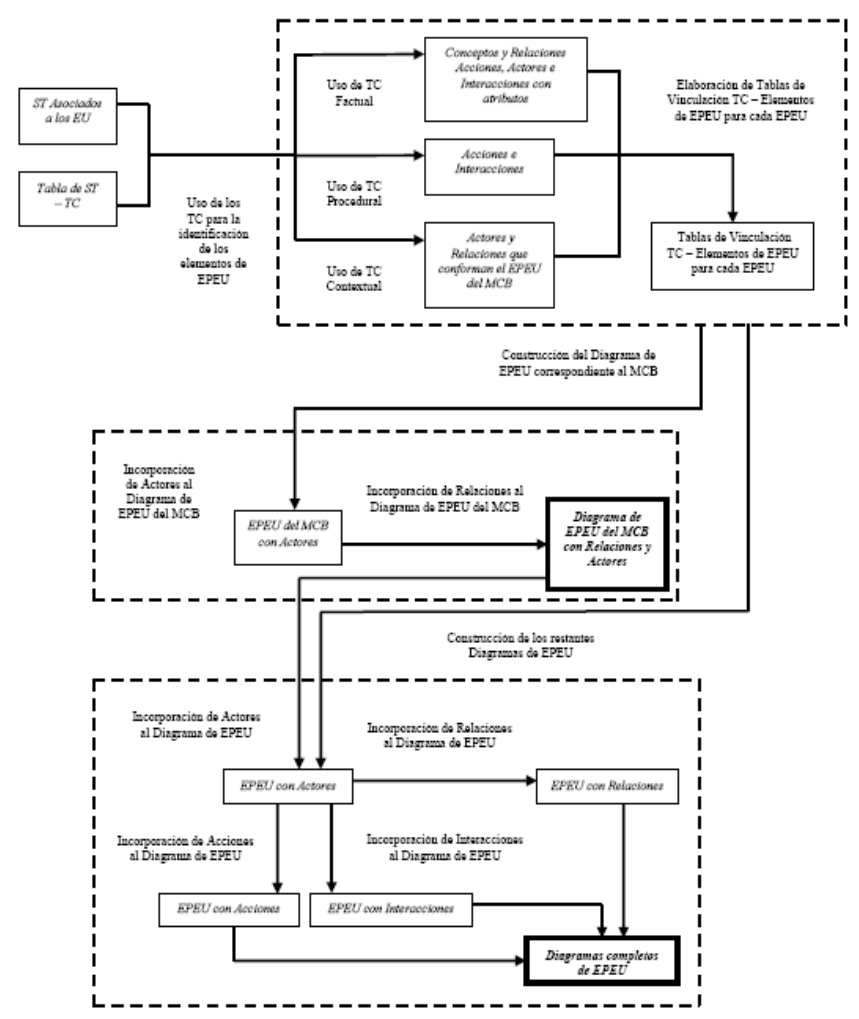

Fig. 11 Esquema y subproductos resultantes de aplicar la Técnica de Construcción del Diagrama de Espacio Problema en Escenarios de Usuario (TCD - EPEU). Nota: por razones de claridad de ilustración no se incluyen los correspondientes subprocedimientos en la Figura 11.

Para comenzar a aplicar la TCD - EU el IR procede a hacer uso de los ST con TC de Asociación y de los diagramas de EPEU, lo que le permite obtener las “Funcionalidades" del problema planteado por el usuario, así como también identificar aquellos actores del EPEU que son necesarios para que el sistema realice estas funcionalidades. Con las funcionalidades y los diagramas de EPEU para los cuales se identificaron estas funcionalidades, el IR confecciona los diagramas correspondientes a los bloques del Espacio Producto de Escenarios de Usuario (EPrEU) para estos EPEU [3], [43], [44], [45].

Finalmente, el IR realiza un proceso de asociación a los efectos de obtener los vínculos existentes entre los elementos de los bloques de EPEU y EPrEU, obteniendo así un único diagrama para cada EU conformado por ambos bloques. Aquellos EU que no contengan funcionalidades asociadas, es 
decir que no posean EPrEU, quedarán conformados por su correspondiente EPEU.

Los diagramas correspondientes a los EU constituyen el producto de salida que proporciona la TCD - EU. La tabla 4 resume los pasos y procedimientos necesarios para la implementación de esta técnica.

\begin{tabular}{|l|}
\hline \multicolumn{1}{|c|}{ Técnica de Construcción del Diagrama de Escenarios de Usuario (TCD - EU) } \\
\hline Entradas: ST con TC de Asociación (de la Tabla ST - TC) y Diagramas de EPEU \\
Salidas: Diagramas de EU \\
\hline Paso 1. Uso del TC de Asociación \\
1.1. Identificación de Funcionalidades \\
1.2. Identificación de Actores necesarios para realizar las funcionalidades \\
1.3. Completar Tablas de Vinculación TC - Elementos de EPEU para los EPEU con \\
TC de Asociación \\
Paso 2. Construcción de los Diagramas de EPrEU para cada EPEU \\
Paso 3. Vinculación de los elementos de los bloques de EPEU y EPrEU para cada EU \\
\hline
\end{tabular}

Tabla 4. Técnica de Construcción del Diagrama de Escenarios de Usuario (TCD - EU)

Paso 1: En este primer paso el IR hace uso del TC de Asociación para la identificación de las funcionalidades del producto software a construir y de los actores que son necesarios para llevar a cabo estas funcionalidades correspondientes al EPEU que se analice. La realización de este paso se lleva a cabo por medio de tres procedimientos, a saber:

i. Identificación de Funcionalidades: por medio de este procedimiento el IR identifica las funcionalidades que debe realizar el sistema, las cuales se encuentran embebidas en aquellos ST en los que se ha identificado TC de Asociación.

ii. Identificación de Actores necesarios para realizar las funcionalidades: por medio de este procedimiento el IR identifica aquellos actores de los EPEU que son necesarios para llevar a cabo las funcionalidades obtenidas en 1.1, los cuales también deben identificarse en los mismos ST con TC de Asociación que se exploraron en " $i$ ".

iii. Completar Tablas de Vinculación TC - Elementos de EPEU para los EPEU con TC de Asociación: por medio de este procedimiento el IR completa aquellas Tablas de Vinculación TC - Elementos de EPEU para los EPEU con TC de Asociación, con las funcionalidades y los actores que se necesitan para su realización.

Por consiguiente, las Tablas de Vinculación TC Elementos de EPEU para los EPEU con TC de Asociación completas con las funcionalidades y actores necesarios para su implementación, constituyen el subproducto de salida correspondiente a este paso.

Paso 2: En este segundo paso, el IR hace uso de las funcionalidades obtenidas (sintetizadas en las Tablas de Vinculación TC - Elementos de EPEU para los EPEU con TC de Asociación) y de los diagramas de EPEU para los cuales se identificaron estas funcionalidades, para confeccionar los diagramas correspondientes a los bloques del Espacio Producto de Escenarios de Usuario (EPrEU) para estos EPEU. Por consiguiente, los Diagramas de EPrEU con las respectivas Funcionalidades constituyen el subproducto de salida de este paso.
Paso 3: En este tercer paso, el IR procede a establecer la "vinculación existente" entre las funcionalidades que conforman cada uno de los diagramas de EPrEU obtenidos en el paso anterior $\mathrm{y}$ aquellos actores pertenecientes al correspondiente EPEU que son necesarios para llevar a cabo estas funcionalidades. Para realizar este paso, el IR hace uso de los bloques de EPEU y EPrEU para cada EU. Desde el punto de vista gráfico, esta "vinculación" consiste en una flecha dirigida desde el actor (alojado en el EPEU) a la funcionalidad (alojada en el EPrEU). Por consiguiente, los Diagramas de EU con sus bloques de EPEU y EPrEU y sus correspondientes vinculaciones constituyen el producto de salida de esta técnica. Cabe señalar, que se tendrán diagramas de EU con los dos bloques correspondientes al EPEU y al EPrEU, y diagramas de EU sin el bloque de EPrEU; es decir, solo con el bloque de EPEU. La técnica propuesta y los subproductos que se obtienen se pueden visualizar en figura 12.

\section{b) Técnica de Refinamiento del Diagrama de} Escenarios de Usuario (TRD - EU)

Por medio de esta técnica se implementa la segunda tarea que debe llevar a cabo el IR en la fase de Análisis Orientado al Producto, denominada Refinamiento de Escenarios de Usuario (REU). Cabe destacar, que el procedimiento que se lleva a cabo para la aplicación de la TRD - EU incluye tanto al IR como al Usuario. Los productos de entrada con que ambos cuentan para aplicar esta técnica son el Discurso de Usuario (DU) original, o también llamado "en crudo", el cual cabe recordar constituye el producto de entrada al "Proceso de Conceptualización" desde la fase misma de Análisis Orientado al Problema, y de cada uno de los Escenarios de Usuario (EU) obtenidos en la tarea anterior. Como producto de salida se obtienen los respectivos Diagramas de Escenarios de Usuario Refinados (EUR).

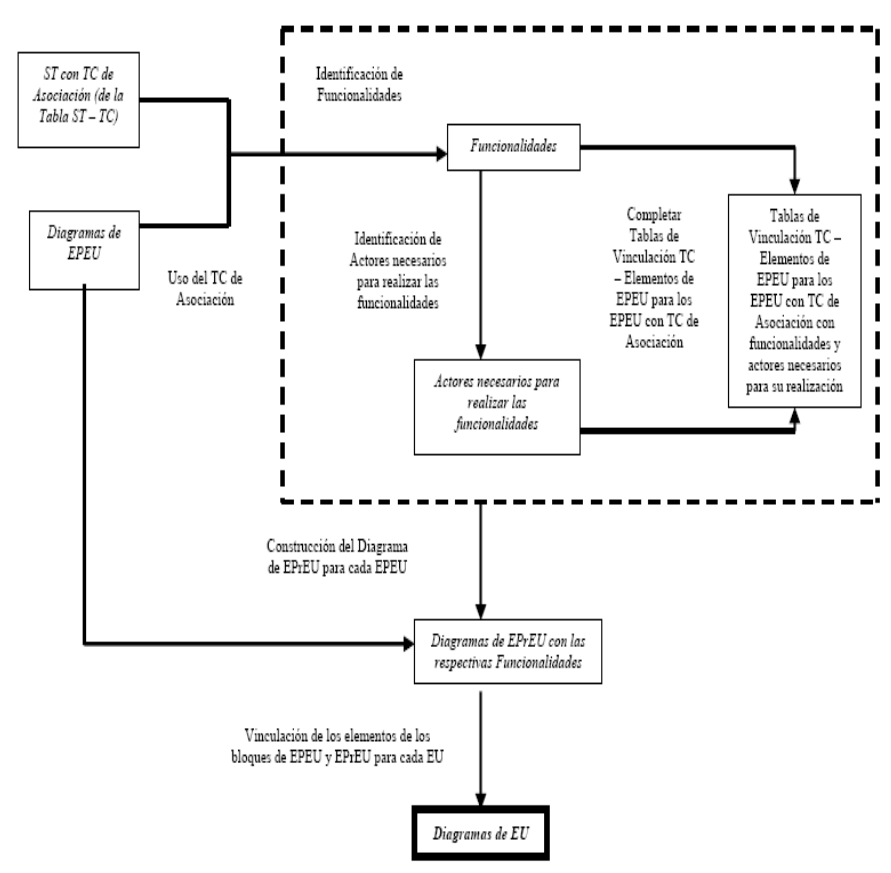

Fig. 12 Esquema y subproductos resultantes de aplicar la Técnica de Construcción del Diagrama de Escenarios de Usuario (TCD - EU)

El procedimiento de aplicación de TRD - EU incluye en primer término una revisión conjunta (Usuario e IR) del 
Discurso de Usuario (DU) original, el cual se lleva a cabo en base a un Análisis de Consistencia del mismo tendiente a identificar inconsistencias, las cuales se clasifican en incompletitudes y contradicciones. Dichas inconsistencias se validan y se depuran para contar con un Discurso de Usuario Refinado (DUR). Estas inconsistencias se ponen de manifiesto en el DU como consecuencia de un lógico "grado de subjetividad" que, muy probablemente, siempre esté presente en el relato del usuario, y que por lo tanto, se ven reflejadas en los EU [3], [20], [46], [47].

A partir de contar con un DU “refinado" (DUR), Usuario e IR realizan un Análisis de Consistencia de los Segmentos de Texto (ST) y Tipos de Conocimiento (TC), el cual consiste en una validación y depuración de los mismos a los efectos de depurar a estos elementos de las inconsistencias provenientes del DU. Luego, Usuario e IR realizan una validación y depuración de los diagramas de EU obtenidos a partir de la aplicación de la técnica TCD - EU, a los efectos de poder contar con diagramas de EUR desprovistos del mayor caudal de subjetividad posible. Como paso final, Usuario e IR efectúan una revisión final de los diagramas de EUR; si ambos consensúan en otorgar grado de conformidad a los mismos se da por concluida la realización de la TRD - EU y se pasa a abordar la próxima técnica del proceso de conceptualización, caso contrario, se comienza nuevamente a aplicar la TRD EU desde el mismo DU original.

Los diagramas correspondientes a los EUR constituyen el producto de salida que proporciona la TRD - EU. La tabla 5 resume los pasos, procedimientos y subprocedimientos necesarios para la implementación de esta técnica.

\begin{tabular}{|l}
\hline Técnica de Refinamiento del Diagrama de Escenarios de Usuario (TRD - EU) \\
Entradas: Discurso de Usuario (DU) y Diagramas de EU \\
Salidas: Diagramas de EUR \\
Paso 1. Análisis de Consistencia del DU \\
1.1. Validación y Depuración de Incompletitudes del DU \\
1.2. Validación y Depuración de Contradicciones del DU \\
1.3. Validación y Depuración del DU \\
Paso 2. Análisis de Consistencia de los ST y TC \\
2.1. Validación y Depuración de los ST \\
2.1.1. Incidencia del DUR en las "frases cortas" \\
2.1.2. Incidencia de las "frases cortas" en los ST \\
2.2. Validación y Depuración de los TC \\
2.2.1. Incidencia de los ST en la identificación del TC Contextual \\
2.2.2. Incidencia de los ST en la identificación del TC Factual \\
2.2.4. Incidencia de los ST en la identificación del TC de Asociación \\
Paso 4. Revisión Final de los Diagramas de EUR \\
Si los Diagramas de EUR son satisfactorios => Fin de la Técnica \\
Si los Diagramas de EUR no son satisfactorios => Volver a Paso 1 \\
Paso 3. Validación y Depuración de los Diagramas de EU \\
3.1. Refinamiento de los Diagramas de EPEU \\
3.2. Refinamiento de los Diagramas de EPrEU \\
3. Obtención de los Diagramas de EUR
\end{tabular}

Tabla 5. Técnica de Refinamiento del Diagrama de Escenarios de Usuario $($ TRD - EU)

Paso 1: En este primer paso, Usuario e IR hacen uso del DU original y realizan el Análisis de Consistencia del DU basado en la identificación de incompletitudes e inconsistencias, para luego obtener un DU "refinado" (DUR). La realización de este paso se lleva a cabo por medio de tres procedimientos, a saber:

i. Validación y Depuración de Incompletitudes: por medio de este procedimiento Usuario e IR detectan información faltante, como por ejemplo, la omisión acerca de la necesidad de validación de las facturas a pagar. Se procede a completar el párrafo correspondiente al DU original con la información faltante.

ii. Validación y Depuración de Contradicciones: por medio de este procedimiento Usuario e IR detectan información contradictoria, como por ejemplo, si en el DU original de establece que las facturas de pago a proveedores deben ser validadas por la tesorería antes de ser abonadas, y en una revisión posterior del discurso, el usuario dice que para que se efectúe el pago de dichas facturas las mismas deben ser validadas por el departamento de compras. Se procede a corregir el párrafo correspondiente al DU original con la información correcta.

iii. Validación y Depuración del DU: por medio de este procedimiento $\mathrm{y}$ con las incompletitudes $\mathrm{y}$ contradicciones depuradas, Usuario e IR obtienen un DU que satisface a ambos. A este DU se lo llama DU "refinado" (DUR).

Por consiguiente, el DU "refinado" (DUR) constituye el subproducto de salida de este paso.

Paso 2: En este segundo paso, Usuario e IR realizan un Análisis de Consistencia de los ST y TC obtenidos a partir del DU original, para lo cual hacen uso del DUR obtenido en el paso anterior y de los ST y TC originales. La realización de este paso se fundamenta en el hecho de que las inconsistencias identificadas en el DU original en los procedimientos " $i$ " $\mathrm{y}$ "ii", seguramente se propagan hacia los ST y TC obtenidos en base a ese DU. La realización de este paso se lleva a cabo por medio de dos procedimientos, y sus correspondientes subprocedimientos, a saber:

i. Validación y Depuración de los ST: por medio de este procedimiento Usuario e IR exploran el grado de impacto que experimentan los ST originales al hacer uso del DUR. Se implementan los siguientes subprocedimientos:

- Incidencia del DUR en las "frases cortas": por medio de este subprocedimiento Usuario e IR analizan la incidencia del DUR obtenido en el Paso 1 en las "frases cortas" obtenidas de la segmentación del DU original. De la aplicación de este subprocedimiento se tienen "frases cortas" nuevas o modificadas, a las que se denomina refinadas.

- Incidencia de las "frases cortas" en los ST: por medio de este subprocedimiento Usuario e IR analizan la incidencia de las "frases cortas" obtenidas en 2.1.1 en los ST originales. De la aplicación de este subprocedimiento se tienen ST nuevos o modificados, a los que se denomina refinados. 
ii. Validación y Depuración de los TC: por medio de este procedimiento Usuario e IR exploran el grado de impacto que experimentan los TC originales, al hacer uso de los ST obtenidos en 2.1.2. Se implementan los siguientes subprocedimientos:

- Incidencia de los ST en la identificación del TC Contextual: por medio de este subprocedimiento Usuario e IR analizan la incidencia de los ST nuevos o modificados obtenidos en el procedimiento del TC Contextual original. De la aplicación de este subprocedimiento se tiene TC Contextual nuevo o modificado, al cual se lo denomina refinado (TC CR).

- Incidencia de los ST en la identificación del TC Factual: por medio de este subprocedimiento Usuario e IR analizan la incidencia de los ST nuevos o modificados obtenidos en el procedimiento 2.1 en el TC Factual original. De la aplicación de este subprocedimiento se tiene TC Factual nuevo o modificado, al cual se lo denomina refinado (TC FR).

- Incidencia de los ST en la identificación del TC Procedural: por medio de este subprocedimiento Usuario e IR analizan la incidencia de los ST nuevos o modificados obtenidos en el procedimiento 2.1 en el TC Procedural original. De la aplicación de este subprocedimiento se tiene TC Procedural nuevo o modificado, al cual se lo denomina refinado (TC PR).

- Incidencia de los ST en la identificación del TC de Asociación: por medio de este subprocedimiento Usuario e IR analizan la incidencia de los ST nuevos o modificados obtenidos en el procedimiento 2.1 en el TC de Asociación original. De la aplicación de este subprocedimiento se tiene TC de Asociación nuevo o modificado, al cual se lo denomina refinado (TC AR).

Por consiguiente, los ST y TC “refinados” (STR) y (TCR) constituyen el subproducto de salida de este paso.

Paso 3: En este tercer paso, Usuario e IR hacen uso de los diagramas de Escenarios de Usuario EU obtenidos a partir del DU original y los STR y los TCR obtenido en el paso anterior, para realizar una validación y depuración de los EU originales. En este sentido, se comienza por validar y depurar los diagramas de EPEU, continuando con los diagramas de EPrEU, para finalmente obtener los diagramas de Escenarios de Usuario Refinados (EUR). La realización de este paso se lleva a cabo por medio de tres procedimientos, a saber:

i. Refinamiento de los Diagramas de EPEU: por medio de este procedimiento Usuario e IR validan y depuran los diagramas correspondientes a los EPEU originales, a través de la inspección de los elementos que los componen (actores, atributos, relaciones, acciones e interacciones). De esta manera, puede darse el caso de tener que agregar o quitar actores, modificar valores de atributos, incluir nuevas interacciones o acciones, etc. Se procede a corregir los diagramas de EPEU originales, obteniendo los correspondientes diagramas de EPEU “refinados” (EPEUR).

ii. Refinamiento de los Diagramas de EPrEU: por medio de este procedimiento Usuario e IR validan y depuran los diagramas correspondientes a los EPrEU originales a través de la inspección de las funcionalidades que los conforman, con los STR, TCR (especialmente el TC de Asociación "refinado" (TC AR)) y los diagramas de EPEUR como soporte, obtenidos en $2.1,2.2$ y 3.1. Se procede a corregir diagramas de EprEU originales, obteniendo los correspondientes diagramas de EPrEU "refinados” (EPrEUR).

iii. Obtención de los Diagramas de EUR: por medio de este procedimiento Usuario e IR validan y depuran las "vinculaciones existentes" entre las funcionalidades que conforman cada uno de los diagramas de EPrEUR obtenidos en 3.2 y los correspondientes actores pertenecientes a cada uno de los diagramas de EPEUR obtenidos en 3.1 que son necesarios para llevar a cabo estas funcionalidades. Se procede a corregir los diagramas de EU originales, obteniendo los correspondientes diagramas de EU "refinados" (EUR).

Por consiguiente, los diagramas de EUR constituyen el subproducto de salida de este paso.

Paso 4: En este cuarto paso, Usuario e IR hacen uso de los diagramas de EUR obtenidos en el paso anterior y realizan una "revisión final" de los mismos contrastándolos con los diagramas de EU que se obtuvieron a partir del DU original. En caso de que Usuario e IR den conformidad a los diagramas de EUR obtenidos, estos constituyen el producto de salida de esta técnica y se da por finalizada la misma pasando a la aplicación de la siguiente, caso contrario se vuelve al Paso 1 y se comienza a aplicar la técnica nuevamente tomando como nuevo punto de partida el último DUR y los últimos diagramas de EU.

El modo de implementación de esta técnica responde a un "ciclo de prototipado evolutivo" [16], [48], [49], mediante el cual los EUR obtenidos se validan frente a los EU originales hasta alcanzar un conjunto de EUR que satisfagan a Usuario e IR, volviendo a ejecutar todos los pasos de la técnica. Esta forma de llevar a cabo el proceso tiene su justificación en el siguiente concepto, propio del espíritu del "ciclo de prototipado evolutivo", el cual establece que los EUR que se van obteniendo en cada ciclo ponen a la luz nuevas inconsistencias en el DU, ya sea en lo que se refiere a aspectos de la realidad del problema como a sus funcionalidades.

Cabe destacar que cada ciclo de aplicación de la técnica, debe llevarse a cabo con el último DUR que se obtuvo del ciclo anterior y los últimos diagramas de EU obtenidos. Este hecho pone en evidencia la importancia que tiene contar con un Discurso de Usuario (DU) "pulido" y "refinado" que refleje de manera fidedigna los requerimientos de usuario, para poder obtener un producto software de de alta calidad. La técnica propuesta y los subproductos que se obtienen se pueden visualizar en figura 13. 
c) Técnica de Construcción del Diagrama del Mapa Unificado de Escenarios de Usuario Refinados (TCD MUEUR)

Por medio de esta técnica se implementa la tercera y última tarea que debe llevar a cabo el IR en la fase de Análisis Orientado al Producto, denominada Construcción del Mapa Unificado de Escenarios de Usuario Refinados (CMUEUR). Para la aplicación de la TCD - MUEUR el IR dispone como productos de entrada de cada uno de los STR (asociados a los EUR) y de los diagramas de EUR, ambos obtenidos a partir de la aplicación de la técnica TRD - EU. Como producto de salida se obtiene el Diagrama de Mapa Unificado de Escenarios de Usuario Refinados (MUEUR).

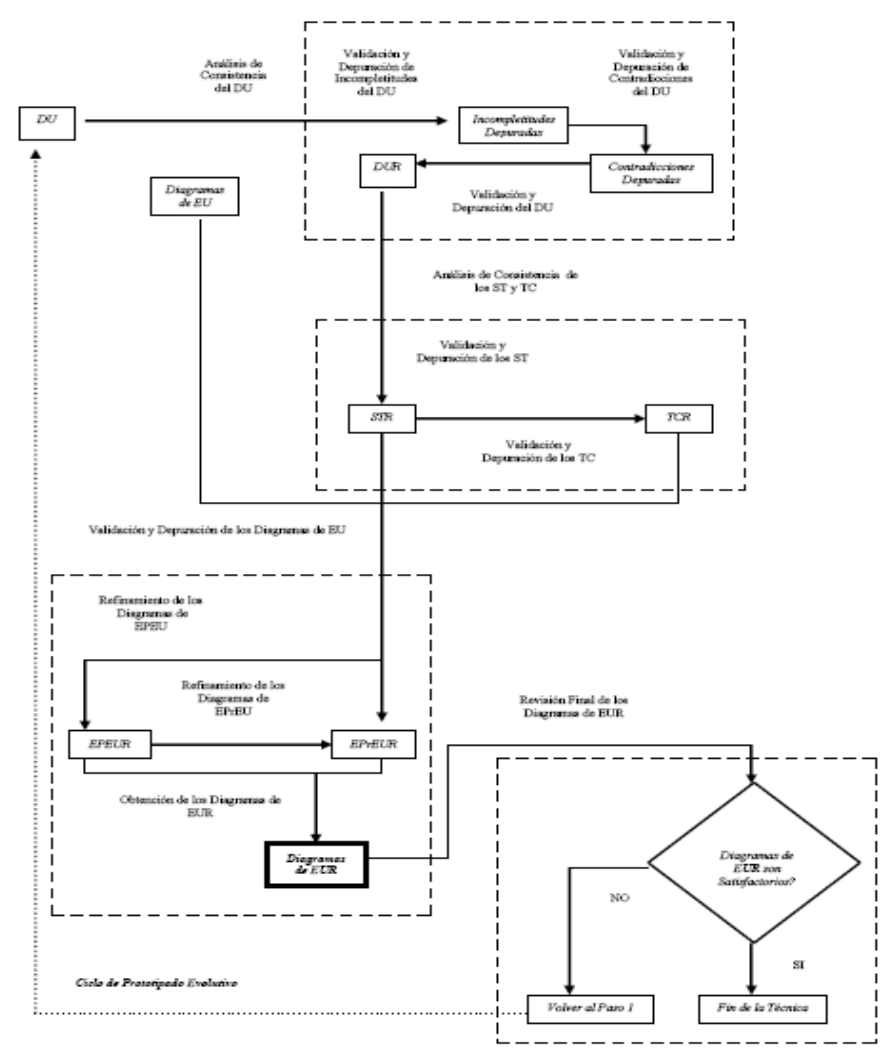

Fig. 13. Esquema y subproductos resultantes de aplicar la Técnica de Refinamiento del Diagrama de Escenarios de Usuario (TRD - EU)

Nota: por razones de claridad de ilustración no se incluyen los correspondientes subprocedimientos en la Figura 13.

Es importante señalar, que un único escenario es representativo de una determinada situación de la realidad con sus funcionalidades asociadas, y en consecuencia, dicho escenario proporciona información parcial sobre el problema y la realidad en la que este se encuentra embebido [17]. Por tal razón, es que resulta sustancial que un análisis basado en escenarios involucre la observación de un conjunto de los mismos a los efectos de identificar aquellos elementos que permiten vincularlos [50], [35], [36]. En tal sentido, la interconexión de escenarios indica una secuencia en la ocurrencia de diversas situaciones que se encuentran embebidas en el Discurso de Escenario de Usuario Refinado (DUR) y que le permiten al IR documentar el "orden temporal" en que tienen lugar los diferentes escenarios obtenidos [34], [38], [51], así como también cuales escenarios son condición necesaria para la ocurrencia de otros (transición entre escenarios); en otros términos, identificar las correspondientes relaciones de precedencia entre escenarios. De esta manera, el diagrama de MUEUR representa una secuencia espacio - temporal acerca de cómo el usuario entiende el problema a resolver y la realidad en la que se encuadra dicho problema. El procedimiento de aplicación de TCD - MUEUR incluye un Análisis de Transición de Escenarios de Usuario Refinados (EUR) mediante el cual se identifican los "Disparadores de Escenarios de Usuario Refinados", los cuales permiten identificar las correspondientes relaciones de precedencia entre EUR. A partir de estos disparadores el IR está en condiciones de establecer los correspondientes vínculos entre EUR que le conducen al Diagrama de MUEUR. El diagrama correspondiente al MUEUR constituye el producto de salida que proporciona esta técnica, la cual se resume en la tabla 6.

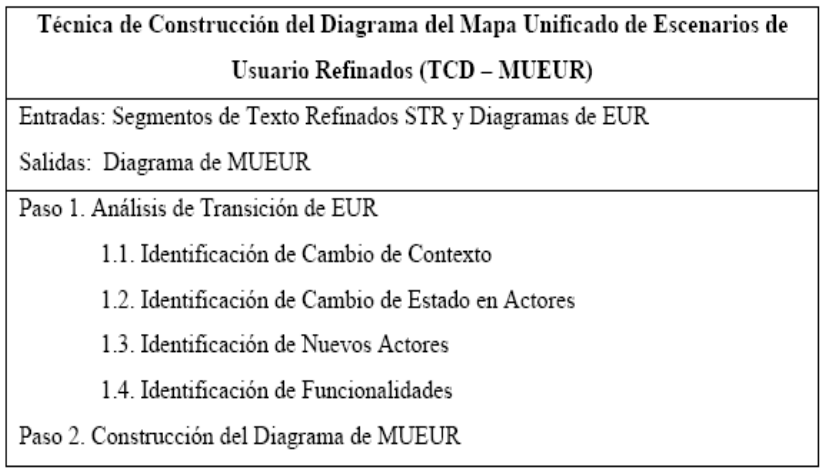

Tabla 6. Técnica de Construcción del Diagrama del Mapa Unificado de Escenarios de Usuario Refinados (TCD - MUEUR)

Paso 1: En este primer paso, el IR identifica los Disparadores de Escenarios de Usuario Refinados (EUR) presentes en los Segmentos de Texto Refinados (STR) y plasmados en los correspondientes EUR. Estos disparadores son considerados como agentes de cambio para cada EUR, ya que producen modificaciones en el cuerpo del escenario estableciendo, de esta manera, aquellos elementos vinculantes entre los mismos que permiten descubrir las correspondientes relaciones de precedencia entre EUR. La realización de este paso se lleva a cabo por medio de cuatro procedimientos de acuerdo a la clase de Disparadores de EUR que identifique el IR, los cuales se deben desarrollar para cada "Transición" entre un EUR y el que le continúa de acuerdo al criterio del IR, partiendo desde como se establece el EUR correspondiente al Marco Contextual Base:

A continuación se especifican cada uno de los cuatro procedimientos que debe llevar a cabo el IR para una "Transición" cualquiera:

i. Identificación de Cambio de Contexto: este disparador se presenta cuando del análisis conjunto de los (STR) y (EUR) el IR identifica un cambio de contexto de la situación que se describe, entendiéndose en tal caso, que se pasa a la descripción de un nuevo EUR. Por ejemplo, si el usuario pasa de describir lo que está sucediendo en el Departamento de Recursos Humanos a describir acontecimientos que tienen lugar en el Departamento de Control de Presupuesto; entonces el IR identifica un cambio de contexto que necesariamente implica una nueva situación, y por 
consiguiente, un nuevo escenario de usuario con nuevos actores y nuevas relaciones. Cuando se describe el Marco Contextual Base (que generalmente corresponde al EUR I), este disparador debe hacer referencia a los actores centrales que componen dicho marco y las correspondientes relaciones entre ellos. En cualquiera de estos casos, cuando se produce un cambio de contexto o cuando se describe el Marco Contextual Base, por lo general el disparador tiene su origen en algún STR del DUR. A este tipo de disparador se lo llama Disparador de EUR Tipo I.

ii. Identificación de Cambio de Estado en Actores: este disparador se presenta cuando del análisis conjunto de los (STR) y (EUR) el IR identifica un cambio de estado en uno o más actores que componen un EUR, entendiendo tal cambio como adición de un nuevo atributo en el actor o cambio de valor de un atributo de este, por lo que se pasa a tener un nuevo EUR con estas modificaciones. Estos cambios pueden producirse a causa de acciones internas de los actores, interacciones entre actores o desde los mismos STR del DUR. Por ejemplo, si en un sistema de reserva de hotel una habitación pasa del estado de "libre" al estado de "reservada", entonces en el actor habitación cambia el valor del atributo disponibilidad de libre a reservada; lo cual origina la transición hacia un nuevo EUR donde pueden tener lugar acciones tales como otorgarle a un pasajero un código de reserva para esa habitación. A este tipo de disparador se lo llama Disparador de EUR Tipo II.

iii. Identificación de Nuevos Actores: este disparador se presenta cuando del análisis conjunto de los (STR) y (EUR) el IR identifica eventos que modifican la composición del EUR actual, como ser el caso del agregado de un nuevo actor. De esta manera, se pasa a tener un nuevo EUR con estas modificaciones. Por lo general, estos cambios se producen a causa de los mismos STR del DUR. Por ejemplo, el ingreso de un nuevo avión en el espacio aéreo de un aeropuerto se puede ver como un evento que origina la transición a un nuevo EUR en un sistema de control de tráfico aéreo. A este tipo de disparador se lo llama Disparador de EUR Tipo III.

iv. Identificación de Funcionalidades: este disparador se presenta cuando del análisis conjunto de los (STR) y (EUR) el IR identifica la presencia de funcionalidades que debe realizar el producto software, modificando la composición del EPrEUR (dado que las funcionalidades se alojan en el Espacio Producto del Escenario de Usuario Refinado) y, en consecuencia, del EUR actual. También es importante identificar aquellos actores que son necesarios para realizar la funcionalidad. Por lo general, estos cambios se producen a causa de los mismos STR del DUR. Por ejemplo, que la organización pretenda llevar una estadística semanal de los clientes que deben dos o más cuotas. La incorporación de esta prestación al Espacio Producto de un determinado EUR origina la transición hacia un nuevo EUR. A este tipo de disparador se lo llama Disparador de EUR Tipo IV. Es importante destacar, que los Disparadores de EUR son originados a causa de hechos correspondientes a la realidad contenida en el DUR; por tal razón, es preciso que cuando el IR identifica un Disparador de EUR también haga referencia a la causa que lo origina, es decir cuál es el hecho de la realidad que da lugar a la presencia de ese disparador. En síntesis, el término disparador engloba tanto al cambio que se produce en el cuerpo del EUR como así también a la causa que lo produce. Por ejemplo, si se produce un cambio en el valor de un atributo de un cierto actor del escenario (disparador tipo II que origina la transición a otro EUR), la causa de este disparador podría ser una interacción de este actor con otro, o si se produce la incorporación de una funcionalidad al espacio producto de un determinado escenario (disparador tipo IV que origina la transición a otro EUR), pudiendo ser la causa de este disparador un cierto párrafo perteneciente a un determinado segmento de texto. Conforme a lo expuesto, es importante documentar un disparador de EUR en función de las características mencionadas y también dependiendo del tipo de disparador.

A continuación se presenta un formato de documentación para cada tipo de EUR, suponiendo una situación cualquiera de transición de escenarios para un sistema determinado y asumiendo que en caso de existir más de un disparador del mismo tipo para la misma transición de un EUR a otro EUR, se los distingue con una apóstrofe; por ejemplo: Disparador de EUR tipo IV (EUR I - EUR II), Disparador de EUR tipo IV (EUR I - EUR II)', Disparador de EUR tipo IV (EUR I - EUR II)', Disparador de EUR tipo IV (EUR I - EUR II)',', y así siguiendo. Cabe aclarar, que para los EUR que figuran entre paréntesis significa que el disparador origina la transición desde el EUR que figura en primer término al EUR que se encuentra en segundo término. Explicado este detalle, se ilustra el formato de representación de disparador para un sistema de reserva de habitaciones en un complejo hotelero:

- Disparador de EUR tipo I (EUR I correspondiente al Marco Contextual Base "Sector de Reservas")

Actores: Gerente de Sector, Jefe de Sector, Formulario de Reserva y Habitación

Relación 1: "Reporta" (entre los Actores Gerente de Sector y Jefe de Sector)

Relación 2: "Controla" (entre los Actores Jefe de Sector y Formulario de Reserva)

- Causa: DUR "STR asociado al EUR I correspondiente al Marco Contextual Base"

- Disparador de EUR tipo II (EUR I - EUR II)

Actor: Formulario de Reserva

Atributo: Procesamiento

Valor en EUR I: Sin procesar

Valor en EUR II: Procesado 
Causa: Interacción "Procesa desde el actor Jefe de Sector al actor Formulario de Reserva" en el EUR II

- Disparador de EUR tipo III (EUR II - EUR III)

Actor que se agrega al EUR III: Pasajero

Atributos: Nombre, Apellido, DNI, Domicilio y $\mathrm{N}^{\circ}$ de Visita

Causa: DUR "STR asociado al EUR III"

- Disparador de EUR tipo II (EUR III - EUR IV)

Actor: Habitación

Atributo: Disponibilidad

Valor en EUR III: Libre

Valor en EUR IV: Reservada

Causa: Interacción "Autoriza Reserva desde el actor

Jefe de Sector al actor Habitación” en el EUR IV

- Disparador de EUR tipo IV (EUR IV - EUR V)

Funcionalidad: Registro mensual sobre la cantidad de veces que se ocupó cada habitación

Actores necesarios para realizar la funcionalidad: Habitación

Causa: DUR "STR asociado al EUR V"

- Disparador de EUR tipo IV (EUR IV - EUR V)'

Funcionalidad: Registro mensual sobre la cantidad de veces que se reservó cada habitación

Actores necesarios para realizar la funcionalidad: Formulario de Reserva

Causa: DUR "STR asociado al EUR V"

Cabe aclarar con respecto a estas dos funcionalidades que se pretenden realizar en un sistema de esta clase, que las mismas se distinguen en el hecho de que una habitación puede ser reservada por un pasajero para un determinado período de tiempo, pero no necesariamente ser ocupada. Por tal razón es aceptable suponer que el Gerente del Sector desee contrastar los registros que se obtengan por medio de estas dos funcionalidades.

Obtenidos los diferentes tipos de Disparadores de EUR por medio de cada uno de los procedimientos de este paso, estos constituyen el subproducto de salida del mismo. De esta manera, el IR está en condiciones de abordar el desarrollo del próximo paso de la técnica.

Paso 2: En este segundo paso, el IR procede a la Construcción del Diagrama de MUEUR, para lo cual se parte de un primer EUR que identifica el Marco Contextual Base (Disparador tipo I), y en el cual se desarrolla la realidad manifestada por el usuario en su discurso. A partir de este primer EUR y con los disparadores tipo II, III y IV identificados en el paso 1, se comienza a elaborar el encadenamiento de los EUR que luego dará lugar al diagrama de MUEUR. En este sentido, el IR debe contemplar que los disparadores pueden presentarse en forma separada o conjunta en un determinado EUR; en otras palabras, la transición de un EUR a otro puede darse a causa de uno o más disparadores, siendo facultad del IR establecer cual o cuales son los disparadores que dan lugar a la transición de un EUR a otro EUR. Por ejemplo, si a partir de un determinado STR asociado a un EUR se identificase el agregado de un nuevo actor $y$ también el cambio en el valor de un atributo de otro actor del mismo EUR (disparadores tipo III y II respectivamente), estos hechos constituirían los dos disparadores que darían lugar al próximo EUR. Por consiguiente, el Diagrama del MUEUR con sus respectivos EUR correctamente "conectados", constituyen el producto de salida de esta técnica, y por consiguiente del proceso de Conceptualización de Requisitos propuesto. La técnica propuesta y los subproductos que se obtienen se pueden visualizar en figura 14.

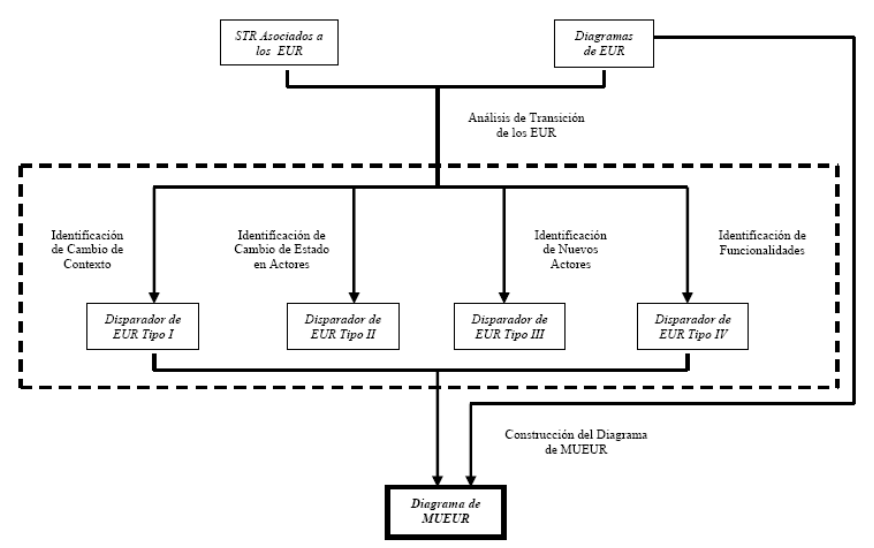

Fig. 14. Esquema y subproductos resultantes de aplicar la Técnica de Construcción del Diagrama del Mapa Unificado de Escenarios de Usuario Refinados (TCD - MUEUR)

\section{CONCLUSIONES}

A continuación se presentan las aportaciones de este trabajo y se destacan las futuras líneas de investigación que se consideran de interés en base al problema abierto que se presenta en este artículo.

\section{A. Aportaciones del trabajo de investigación}

En este trabajo se ha corroborado que se pueden plantear distinciones en el discurso del usuario que permitan diferenciar sub-dominios de análisis que minimicen la brecha conceptual entre la educción de requisitos y el modelado conceptual. Estos sub-dominios son los relacionados con: [a] la descripción de los componentes de la realidad del ambiente de trabajo del usuario que su discurso pone en evidencia; $y$ [b] los aspectos relacionados con las funcionalidades que el usuario espera que el artefacto software posea.

En este contexto, este artículo ha propuesto:

- Un modelo de proceso de conceptualización de requisitos que se desarrolla en dos fases: una de Análisis Orientado al Problema y la otra de Análisis Orientado al Producto.

- Para la Fase de Análisis Orientado al Problema, se han propuesto las siguientes tareas: [i] Segmentación del Discurso de Usuario, la cual necesita del Discurso de Usuario como producto de entrada y proporciona como producto de salida los correspondientes Segmentos de Texto, [ii] Análisis Cognitivo de los Segmentos de Texto, que toma como producto de entrada a los Segmentos de Texto y proporciona como producto de salida los Tipos de Conocimiento embebidos en estos segmentos; y [iii] Construcción del Espacio Problema en Escenarios de Usuario, que tiene como insumos a los Tipos de Conocimiento y a los Segmentos de Texto, y proporciona como producto de salida los correspondientes Diagramas de Espacio Problema en Escenarios de Usuario.

- Para la Fase de Análisis Orientado al Producto, se han propuesto las siguientes tareas: [iv] Construcción de Escenarios de Usuario, la cual necesita como 
productos de entrada a los Segmentos de Texto con Tipo de Conocimiento de Asociación y los Espacio Problema en Escenarios de Usuario, los cuales se procesan en el desarrollo de esta tarea y se obtienen los respectivos Escenarios de Usuario (EU); [v] Refinamiento de Escenarios de Usuario, que tiene como insumos a los Escenarios de Usuario y al Discurso de Usuario, que proporciona como producto de salida los correspondientes Escenarios de Usuario Refinados y [vi] Construcción del Mapa Unificado de Escenarios de Usuario Refinados que tiene como insumos los Escenarios de Usuario Refinados y los Segmentos de Texto Refinados (STR) para producir el Mapa Unificado de Escenarios de Usuario Refinados.

- Para la Fase de Análisis Orientado al Problema, se han desarrollado las técnicas: Técnica de Segmentación del Discurso de Usuario, Técnicas Cognitivas de Identificación de Conocimientos Factuales, Procedurales, Contextuales y de Asociación y la Técnica de Construcción del Diagrama de Espacio Problema de Escenarios de Usuario.

- Para la Fase de Análisis Orientado al Producto, se han desarrollado las técnicas: Técnica de Construcción del Diagrama de Escenarios de Usuario, Técnica de Refinamiento del Diagrama de Escenarios de Usuario y Técnica de Construcción del Diagrama del Mapa Unificado de Escenarios de Usuario Refinado.

- La propuesta de modelo de proceso de conceptualización de requisitos, las tareas y técnicas asociadas han sido validadas en dos dominios de conocimiento con características bien diferenciadas: el primero sobre un Sistema de Abastecimiento de Combustible de Aeronaves en el Contexto de las Operaciones Aeroportuarias, el cual se circunscribe en el dominio de los sistemas de información clásicos; y el segundo correspondiente a un Sistema de Operaciones Bancarias por Cajero Automático, el cual se circunscribe dentro de los sistemas de información transaccional.

\section{B. Futuras líneas de investigación}

Durante el desarrollo de este proyecto han surgido cuestiones que si bien no son centrales al tema abordado en la misma, constituyen temas concomitantes que (en opinión de quien escribe) darían lugar a las siguientes líneas de investigación futuras:

- En este trabajo se han utilizado técnicas de Análisis Cognitivo y técnicas de Ingeniería de Conocimiento. Estos conocimientos no forman parte de la los estándares fijados para la disciplina con lo que cabe preguntarse:

- ¿Qué conocimiento debería tener el ingeniero de requisitos para poder realizar conceptualización de requisitos?

- ¿Debería buscarse una formación interdisciplinaria?

- ¿Qué disciplinas deberían estar involucradas?

- El proceso de conceptualización de requisitos suele ser subestimado al momento de asignar tiempos y recursos en la correspondiente planificación y presupuestación del proyecto. Poder concebir un proceso con fases y tareas como las propuestas en este artículo acerca posiciones respecto de disponer de objetos conceptuales a los que asignarle recursos y prever su desarrollo en una línea de tiempo. En este contexto, se plantea el interés de trabajar en el desarrollo de herramientas que permitan estimar el esfuerzo que llevaría realizar este proceso de conceptualización.

- $\mathrm{Si}$ bien el proceso propuesto en este trabajo aporta sistematicidad al proceso de conceptualización de requisitos y el mismo ha sido validado en dominios representativos, quedan como temas de trabajo abiertos:

- La validación empírica más amplia del proceso de conceptualización de requisitos mediante la técnica de muestras apareadas basadas en grupos experimental y de control.

- La validación empírica de las técnicas propuestas en un conjunto vasto y representativo de dominios de aplicación (sistemas de tiempo real entre otros).

- A los efectos de poder obtener modelos conceptuales de "alta calidad", entre los cuales se pueden citar: diagramas de casos de uso, diagramas de clases, diagramas de objetos, diagramas de interacción (secuencia $\mathrm{y}$ colaboración) y diagramas de estado, entre otros; seria aconsejable considerar una línea de investigación orientada a estudiar como se derivan estos modelos a partir de los diferentes productos que proporciona el proceso propuesto en este trabajo.

\section{REFERENCIAS}

[1] Alford, M. 1977. A Requirements Engineering Methodology for Real-Time Processing Requirements. IEEE Transactions on Software Engineering, SE-3(1).

[2] Yeh, R., Zave, P. 1980. Specifiying Software Requirements, Proc. of the IEEE, 68(9): 1077-1085.

[3] Davis, A. 1993. Software Requirements: Objects, Functions and States; Prentice-Hall International.

[4] Chen, P. 1990. Entity-relationship Approach to Data Modeling. In Systemand Software Requirements Engineering, Thayer RH, Dorfman M (eds). IEEE. Computer Society Press.

[5] Juristo, N. 1991. Método de construcción del núcleo de una base de conocimientos a partir de un modelo de clasificación documental. Tesis Doctoral, Universidad Politécnica de Madrid.

[6] Newell, A., y Simon, H. 1972. Human Problem Solving. Englewood Cliffs, NJ: Prentice Hall.

[7] Gómez, A., N. Juristo, C. Montes, J. Pazos, Ingeniería del Conocimiento, Ed. Centro de Estudios Ramón Areces, (1997).

[8] García Martínez, R. y Britos, P. 2004. Ingeniería de Sistemas Expertos. Editorial Nueva Librería. ISBN 987-1104-15-4.

[9] Kotonya, G., and Sommerville, I. 1998. Requirements Engineering: Processes and Techniques. John Wiley and Sons.

[10] Faulk, S. 1997. Software Requirements: A Tutorial; In Software Engineering, IEEE Computer Society Press, pp 82-101.

[11] Blum, B.I. Beyond Programming: To a New Era of Design. Oxford University Press, 1996.

[12] Wieringa, R. 1995. Requirements Engineering: Frameworks for Understanding; John Wiley.

[13] Beringer, D. 1995. Model Architecture Frame: Quality Management in a Multi Method Environment; Proceedings of the SQM'95.

[14] Van Griethuysen, J.J.; ISO - Concepts and Terminology for the Conceptual Schema and the Information Base; N695, ISO/TC9/SC5/WG3, 1982. 
[15] Chatzoglou P., Soteriou A. 1999. A DEA framework to assess the efficiency of the software requirements capture and analysis process. Decision-Sciences. 30(2): 503-31.

[16] Sommerville, I. 2005. Ingeniería de Software, Addison-Wesley.

[17] Robertson S. 2002. Project Sociology: Identifying and involving the stakeholders, ICFAI University Press.

[18] Christel, M. and Kang, K. 1992. Issues in Requirements Elicitation. Software Engineering Institute Technical Report CMU/SEI-92-TR-12. Carnegie Mellon University.

[19] Van der Vos, B., Gulla, J., Van de Riet, R., 1995. Verification of Conceptual Models based in Linguistic Knowledge. NLDB 1995

[20] [Loucopoulos, P., Karakostas, V. 1995. System Requirements Engineering. McGraw-Hill.

[21] Zave, P. 1990. A Comparison of the Major Approaches to Software Specification and Design. In System and Software Requirements Engineering, Thayer RH, Dorfman M (eds). IEEE. Computer Society Press.

[22] Kaindl, H. 1999. Difficulties in the transition from OO analysis to design. IEEE Software, 16(5).

[23] Sutcliffe, A., Maiden, N. 1992. Analysing the Novice Analyst: Cognitive Models in Software Engineering; International Journal of Man-Machine Studies, 36(5).

[24] Yu, E., Mylopoulos, J. 1994. Uderstanding "Why" in Software Process Modelling, Analysis and Design; Proceedings of the 16th International Conference on Software Engineering.

[25] Holtzblatt, K., and H. Beyer. Requirements Gathering: The Human Factor. Communications of the ACM, 38, 5 (May 1995), pp. 31-32.

[26] Beringer, D. 1996. The Goals of the Analysis Model; Technical Report 96/216.

[27] Jalote, P. 1997. An Integrated Approach to Software Engineering; Springer-Verlag.

[28] Juristo, N., Moreno, A. 2000. Introductory paper: Reflections on Conceptual Modeling. Data and Knowledge Engineering, 33(2): 103-117

[29] Davis, A. and Hickey, A. 2003. Requirements Elicitation and Requirements Elicitation Technique Selection: A Model of Two Knowledge-Intensive Software Development Processes. Proceedings of the Thirty-Sixth Hawaii International Conference on System Sciences, Los Alamitos, California: IEEE Computer Society Press.

[30] Robertson, S., Robertson, J. 1999. Mastering the Requirements Process. Addison-Wesley.

[31] Booch, G.; Object-Oriented Design with Applications; Benjamin Cummings, 1991.

[32] Jacobson, I; Object-Oriented Software Engineering: a Use Case Approach; Addison-Wesley, 1992.

[33] Potts C. Using schematic scenarios to understand user needs. In: Proceedings of DIS'95 - Symposium on designing interactive systems: processes, practices and techniques. ACM Press/ University of Michigan, 1995, pp 247-256.

[34] Carroll, J. 1995. Introduction: The Scenario Perspective on System Development, en "Scenario- Based Design: Envisioning Work and Technology in System Development", editor J. Carroll John Wiley \& Sons.

[35] Zorman L. Requirements envisaging by utilizing scenarios (Rebus). PhD dissertation, University of Southern California, 1995.

[36] Leite, J., Hadad, G., Doorn, J., Kaplan, G. 2000. A Scenario Construction Process, Requirements Engineering Journal, Vol.5(1): 38-61.
[37] Breitman KK, Leite JCSP. A framework for scenario evolution. In: Proceedings of the IEEE international conference on requirements engineering. IEEE Computer Society Press, Los Alamitos, CA, 1998, pp 214-221.

[38] Hadad, G., Kaplan, G., Oliveros, A., Leite, J. 1997. Construcción de Escenarios a partir del Léxico Extendido del Lenguaje. Proceedings del Simposio de Tecnología de Software. XXVI JAIIO. Pág. 65-77.

[39] Manilow, A. 2005. Teaching proportion word problems using a multiple linked-representational software design - Columbia University doctoral dissertation.

[40] Anderson, J. 2006. Cognitive Psychology and its implications Watson Guptill Publications.

[41] Black. J. 1992. Types of Knowledge Representation CCTE Report.Teachers College. Columbia University Press.

[42] Hossian, A. 2003. Sistema de Asistencia a la Selección de Estrategias y Actividades Instruccionales. Tesis de Master en Ingeniería de Software - Universidad Politécnica de Madrid, Madrid.

http://www.iidia.com.ar/rgm/tesistas/hossiantesisdemagister.pdf. Pagina vigente al 14/01/2012.

[43] Potts C., K. Takahashi, A.I. Anton, Inquiry-based requirements analysis. In IEEE Software 11(2), pp. 21-32, 1994.

[44] Rolland, C., Souveyet, C, Ben Achour, C.: Guiding Goal Modeling Using Scenarios, IEEE Transactions on Software Engineering, Vol. 24, N 12, 1998, pp. $1055-1071$.

[45] Rolland C., Grosz G., Kla R., Experience With Goal-Scenario Coupling In Requirements

[46] [Kavakli E., Loucopoulos P., Filippidou D., Using Scenarios to Systematically Support Goal- Directed Elaboration for Information System Requirements, IEEE Symposium and Workshop on Engineering of Computer Based Systems (ECBS'96), 1996, GERMANY.

[47] Leite, J., Rossi, G., Balaguer, F., Maiorana, V., Kaplan, G., Hadad, G., Oliveros, A. 1997. Enhancing a Requirements Baseline with Scenarios. Proceedings of IEEE Third International Requirements Engineering Symposium, IEEE Computer Society Press. Pàg. 44-53.

[48] Pressman, R. S. 2001. Ingeniería del Software: Un enfoque práctico. 3 ed. McGraw-Hill, Madrid. Reigeluth, C. M. 1999. Instructional design theories and models: a new paradigm of instructional theory. Lawrence Erlbaum Associates Publishers. Washington. USA

[49] [Böehm, B. W. A Spiral Model of Software Development and Enhancement. Computer, May 1988, pp. 61-72.

[50] Booch, G.: Scenarios, Report on Object Analysis and Design, Vol. 1, No 3, 1994, pp. 3-6.

[51] Hossian, A., Sierra, E., Britos, P., Ochoa, A., García-Martínez, R. 2007. Hacia una Metodología Orientada al Conocimiento para la Educción de Requisitos en Ingeniería del Software. Proceedings VI Ibero-American Symposium on Software Engineering: 107-114.

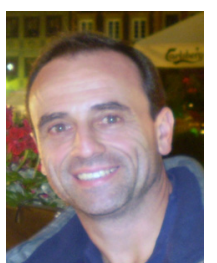

Alejandro Hossian. Ingeniero Civil (Universidad Católica Argentina). Máster en Ingeniería de Software (Universidad Politécnica de Madrid).. Especializado en Sistemas de expertos - Instituto Técnico Buenos Aires (ITBA). Doctor en Ciencias Informáticas, Universidad Nacional de La Plata. Profesor de la Universidad Tecnológica Nacional - Facultad Regional Neuquén (UTN - FRN). Director del Grupo de de Investigación en Sistemas Inteligentes Aplicados a Ingeniería. Sus temas de interés incluyen: robótica, redes neuronales artificiales e Inteligencia Artificial. 